\title{
On the convergence of the best-response algorithm in routing games
}

\author{
O. Brun ${ }^{1,2}$, B.J. Prabhu ${ }^{1,2}$ and T. Seregina ${ }^{1,3}$ \\ ${ }^{1}$ CNRS, LAAS, 7 avenue du colonel Roche, F-31400 Toulouse, France \\ ${ }^{2}$ Univ de Toulouse, LAAS, F-31400 Toulouse, France \\ ${ }^{3}$ Univ de Toulouse, INSA, F-31400 Toulouse, France \\ \{brun, balakrishna.prabhu, tseregin\}@laas.fr
}

\begin{abstract}
We investigate the convergence of sequential best-response dynamics in a routing game over parallel links. Each player controls a nonnegligible portion of the total traffic, and seeks to split its flow over the links of the network so as to minimize its own cost. We prove that best-response operators are lipschitz continuous, which implies that a sufficient condition for the convergence of the best-response dynamics is that the joint spectral radius of Jacobian matrices of best-response operators be strictly less than unity. We establish the specific structure of these Jacobian matrices for our game, and show that this condition is met in two cases: (a) two-player game for an arbitrary number of links and for a wide class of cost functions; and (b) for arbitrary numbers of players and links in the case of linear latency functions. For latency functions satisfying reasonable convexity assumptions, we conjecture that the proposed sufficient condition is met for arbitrary numbers of players and links.
\end{abstract}

\section{Introduction}

Game theory has emerged as a fundamental tool for the design and analysis of decentralized resource allocation mechanisms in networks. It has found applications in as diverse areas as load-balancing in server farms [5, 14, 12, 20, power control and spectrum allocation in wireless networks [11, 26, 23], or congestion control in the Internet [1, 18, 24, 33.

In recent years, substantial research effort has been devoted to the study of non-cooperative routing games in which each origin/destination flow is controlled by an autonomous agent that decides how its own traffic is routed through the network (cf. [3, 13, 21, 28] and reference therein). Apart from the gain in scalability with respect to a centralized routing, there are wide-ranging advantages to such a decentralized routing scheme, including ease of deployment and robusteness to failures and environmental disturbances. However, several questions arise when seeking to design and implement such a non-cooperative routing scheme.

One of the most studied one pertains to the inefficiency of non-cooperative routing mechanisms. Indeed, in general, the Nash equilibrium resulting from the interactions of many self-interested agents does not correspond to an optimal routing solution. Numerous works have therefore focused on obtaining performance guarantees for non-cooperative routing schemes [32, 30, 31, 35, 4, 10. This is usually done by evaluating the Price of Anarchy, a standard measure of the inefficiency of decentralized algorithms introduced by Koutsoupias and Papadimitriou 22. A small value of the Price of Anarchy indicates that, in the worst case, the gap between a Nash Equilibrium and the optimal solution is not significant, and thus that good performances can be achieved even without a centralized control.

In this work, we address a different question: do uncoordinated routing agents converge to a Nash equilibrium? Thus, rather than the quality of the resulting routing strategy, we are concerned with the convergence of autonomous routing agents to a Nash equilibrium under some "natural" dynamics. More precisely, we address this question assuming the well-known (myopic) best-response dynamics. Best-response dynamics play a central role in game theory [8]. For instance, the Nash equilibrium concept is implicitly based on the assumption that players follow best-response dynamics until they reach a state from which no player can improve his utility. In a game, the best-response of player is defined as its optimal strategy 
conditioned on the strategies of the other players. It is, as the name suggests, the best response that the player can give for a given strategy of the others. Best-response dynamics then consists of players taking turns in some order to adapt their strategy based on the most recent known strategy of the others (without considering the effect on future play in the game). In this paper, we will restrict ourselves to the sequential (or round robin) best-response dynamics, where players play in a cyclic manner according to a pre-defined order.

The focus of this paper is the convergence of sequential best-response dynamics in a network of parallel links, shared by a finite number of selfish users. Each user controls a nonnegligible portion of the total traffic, and seeks to split his flow over the links of the network so as to minimize his own cost. This model was introduced in the seminal paper of Orda et al. 29, where it shown that there exists a unique Nash equilibrium under reasonable convexity assumptions on the edge latency functions. The users may have different traffic demands. When all users control the same amount of traffic, the convergence to the Nash equilibrium follows from the fact that the symmetric game is a potential game, that is, the Nash equilibrium corresponds to the minimum of a convex optimization problem [13. For the asymmetric game, convergence results are available only in some special cases. In [29, the convergence to the unique Nash equilibrium of the two-player routing game was proved when there are only two parallel links. As pointed out by the authors, the convergence proof is not readily extendible to more general cases. Altman et al. also study the two-link case 22. Assuming linear latency functions for the links, they prove the convergence of the sequential best-response dynamics for any number of players. More recently, Mertzios has proven that, for the large class of edge latency functions introduced in 29, the two-player routing game converges to the unique Nash equilibrium in a logarithmic number of steps [27. His proof of convergence relies on a potentialbased argument. Namely, he shows that the amount of flow that is reallocated in the network at each step is strictly decreasing. Unfortunately, this argument does not seem to readily extend to more than two players. We also refer to [19, 17, 16] for convergence results on related, but different, problems.

Contributions: We propose a different approach to study of the convergence of best-response dynamics. The key idea to prove the convergence is to study the Jacobian matrices of best-response functions, and to analyze how long products of such matrices grow as a function of the number of best-response updates. One of the most prominent quantities characterizing the growth rate of matrix products is the so-called joint or generalized spectral radius. We show that the best-response function is Lipschitz, and establish the specific structure of their Jacobian matrices for our game. Then, a sufficient condition for the convergence of the best-response dynamics is that their joint spectral radius be strictly less than unity. We thus obtain a purely structural sufficient condition that allows to reduce the analysis of the convergence of the sequential best-response dynamics to the analysis of the joint spectral radius of certain matrices. This condition is used to prove the convergence of the two-player game for an arbitrary number of links. We also prove the convergence to the Nash equilibrium for arbitrary numbers of players and links in the case of linear latency functions. Furthermore, although we were not able to prove it, we conjecture that the proposed sufficient condition is valid for any numbers of players and links.

The paper is organized as follows. In Section 2, we describe the non-cooperative routing game under investigation and introduce best-response dynamics as well as some notations. In Section 3, we outline the non-linear spectral radius approach to convergence, and present several properties of the best-response function, and compute the structure of its Jacobian matrix. In Section 4 we state our main result, and prove the convergence of the best-response function for the two-player game with general cost functions and of the $K$-player game with linear cost functions.

\section{Problem statement}

\section{$2.1 \quad$ Notations}

In the following, $\mathbb{R}_{+}$denotes the set of non-negative real numbers. Recall that the 1-norm of a vector $\mathbf{x} \in \mathbb{R}^{S}$ is $\|\mathbf{x}\|_{1}=\sum_{i=1}^{S}\left|x_{i}\right|$. For $\mathbf{x} \in \mathcal{X}, \mathcal{B}_{o}(\mathbf{x}, r)$ will denote the open ball of radius $r$ centered at point $\mathbf{x}$, i.e., $\mathcal{B}_{o}(\mathbf{x}, r)=\left\{\mathbf{z} \in \mathcal{X}:\|\mathbf{x}-\mathbf{z}\|_{1}<r\right\}$. Let $\mathbf{1}$ denote the column vector $(1,1, \ldots, 1)^{T}$.

We let $I$ and 0 denote the identity and the zero matrices, respectively (their sizes will be clear from the context). A matrix $A$ is positive, and we write $A \geq 0$, if and only if $a_{i, j} \geq 0, \forall i, j$, and that it is negative 
if $-A$ is positive. We recall that the 1 -norm of a matrix $A$ is $\|A\|_{1}=\max _{j} \sum_{i}\left|a_{i j}\right|$. denote by $\sigma(A)$ the spectrum of the matrix $A$, i.e., $\sigma(A)=\{\lambda \in \mathbb{R}: \exists \mathbf{x} \neq 0, A \mathbf{x}=\lambda \mathbf{x}\}$, by $\rho(A)=\max _{\lambda \in \sigma(A)}|\lambda|$ its spectral radius, and we recall that $\rho(A) \leq\|A\|_{1}$. If $A_{1}, \ldots, A_{n}$ is a collection of matrices, we denote by $\prod_{i=1}^{n} A_{i}$ the product $A_{n} A_{n-1} \ldots A_{1}$.

For any function $f$ that is differentiable at point $\mathbf{x}$, we denote by $\mathrm{D} f(\mathbf{x})$ its Jacobian matrix at $\mathbf{x}$.

\subsection{Non-cooperative routing game}

We investigate a non-cooperative routing game with $K$ routing agents and $S$ links in which each routing agent can control how its own traffic is routed over the parallel links. This routing game is depicted on Figure 1

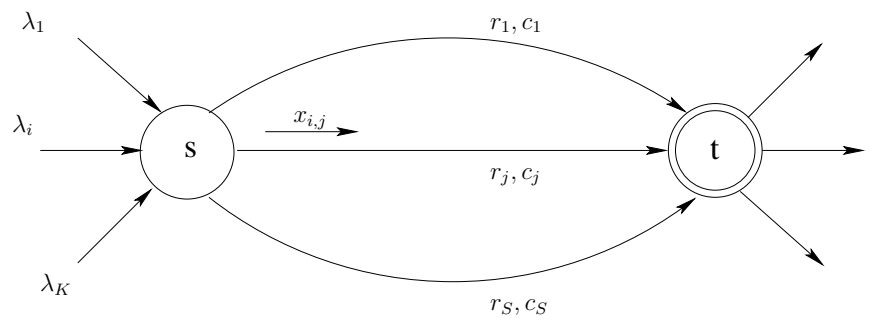

Figure 1: Traffic classes route their packets over parallel links.

Denote by $\mathcal{S}=\{1, \ldots, S\}$ the set of links. Link $j \in \mathcal{S}$ has capacity $r_{j}$ and a holding cost $c_{j}$ per unit time is incurred for each packet sent on this link. We let $\pi_{j}=c_{j} / r_{j}$ denote the cost per unit capacity for link $j$.

We let $\mathcal{C}=\{1, \ldots, K\}$ be the set of routing agent and $\lambda_{i}$ be the traffic intensity of routing agent $i$. We shall also refer to routing agent $i$ as traffic class $i$, or user $i$. Each class can control how its own traffic is splitted over the parallel links and seeks to minimize its own cost. Let $\mathbf{x}_{i}=\left(x_{i, j}\right)_{j \in \mathcal{S}}$ denote the routing strategy of class $i$, with $x_{i, j}$ being the amount of traffic it sends over link $j$. We let $\mathcal{X}_{i}$ denote the set of routing strategies for class $i$, i.e., the set of vectors $\mathbf{x}_{i} \in \mathbb{R}^{S}$ such that $0 \leq x_{i, j}<r_{j}$ for all $j \in \mathcal{S}$, and $\sum_{j \in \mathcal{S}} x_{i, j}=\lambda_{i}$

A strategy profile is a choice of a routing strategy for each user such that the stability condition $\sum_{i \in \mathcal{C}} x_{i, j}<r_{j}$ is satisfied for all links $j \in \mathcal{S}$. It is thus a vector $\mathbf{x}=\left(\mathbf{x}_{i}\right)_{i \in \mathcal{C}}$ belonging to the product strategy space $\bigotimes_{i \in \mathcal{C}} \mathcal{X}_{i}$ such that $\sum_{i \in \mathcal{C}} x_{i, j}<r_{j}$, for all $j \in \mathcal{S}$. It will be assumed throughout the paper that $\sum_{i \in \mathcal{C}} \lambda_{i}<\sum_{j \in \mathcal{S}} r_{j}$, so that $\mathcal{X} \neq \emptyset$.

The optimization problem solved by class $i$, which depends on the routing decisions of the other classes, can be formulated as follows:

$$
\begin{aligned}
& \underset{\text { minimize }}{\min } T_{i}\left(\mathbf{x} \mathbf{x}_{-i}\right)=\sum_{j \in \mathcal{S}} \pi_{j} x_{i, j} \phi\left(\rho_{j}\right) \\
& \text { subject to } \\
& \qquad \begin{array}{l}
\mathbf{x} \in \mathcal{X}_{i}, \\
y_{j}=x_{i, j}+\sum_{k \neq i} x_{k, j}, \quad \forall j \in \mathcal{S}, \\
\rho_{j}=y_{j} / r_{j}, \quad \forall j \in \mathcal{S}, \\
\rho_{j}<1, \quad \forall j \in \mathcal{S},
\end{array}
\end{aligned}
$$

In the above formulation, $y_{j}$ represents the total traffic offered to link $j, \rho_{j}$ is the utilization rate of this link, and $\phi$ is the cost associated to the link when there is a traffic of $y_{j}$ flowing through it. In transportation or communication networks, $\phi$ models the delay on the road or the link. The total cost incurred by user $i$ is then the sum of the cost of individual links weighted by the amount of traffic the user sends on each 
of the links. Thus, given the strategies of the others, user $i$ seeks to minimize its total cost subject to flow conservation and stability constraints.

Assumption 1 We shall make the following assumptions on the cost function $\phi$ :

$\left(A_{1}\right) \phi:[0,1) \rightarrow[0, \infty)$,

$\left(A_{2}\right) \lim _{\rho \rightarrow 1^{-}} \phi(\rho)=+\infty$,

$\left(A_{3}\right)$ continuous, strictly increasing, convex function, and is twice continuously differentiable.

Remark 1 At first glance, it appears that the assumptions are not loose enough to include polynomial cost

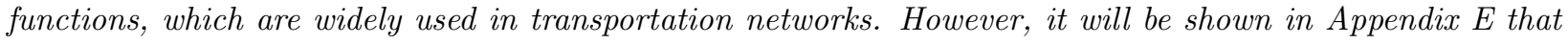
any function satisfying

$\left(B_{1}\right) \phi:[0, \infty) \rightarrow[0, \infty)$,

$\left(B_{2}\right) \lim _{\rho \rightarrow \infty} \phi(\rho)=+\infty$, and

$\left(B_{3}\right)\left(A_{3}\right)$,

has an equivalent function which satisfies assumptions $\left(A_{1}\right)-\left(A_{3}\right)$. Two functions are said to be equivalent if the solution of (BR-i) with one function is also the solution of (BR-i) with the other. Thus, results obtained for functions satisfying $\left(A_{1}\right)-\left(A_{3}\right)$ will be applicable to functions that satisfy $\left(B_{1}\right)-\left(B_{3}\right)$.

We note that Problem (BR-i) is well-defined for all points $\mathbf{x} \in \mathcal{X}$ since $\sum_{k \neq i} x_{k, j}<r_{j}$ for all links $j$.

\subsection{Nash equilibrium}

A Nash equilibrium of the routing game is a strategy profile from which no class finds it beneficial to deviate unilaterally. Hence, $\mathbf{x}^{*} \in \mathcal{X}$ is a Nash Equilibrium Point (NEP) if $\mathbf{x}_{i}^{*}$ is an optimal solution of problem (BR-i) for all classes $i \in \mathcal{C}$, that is, if

$$
\mathbf{x}_{i}^{*}=\arg \min _{\mathbf{z} \in \mathcal{X}_{i}} T_{i}\left(\mathbf{z}, \mathbf{x}_{-i}^{*}\right), \quad \forall i \in \mathcal{C},
$$

where $\mathbf{x}_{-i}^{*}$ is the vector of strategies of all players other than player $i$ at the NEP.

It follows from our assumptions on the function $\phi$, that the link cost functions are a special case of type-B functions, as defined in reference [29. As proved in Theorem 2.1 of this reference, this implies the existence of a unique NEP for our routing game. In the following, we shall denote by $\mathbf{x}^{*}$ this Nash equilibrium point.

\subsection{Best response dynamics}

The best-response of player is defined as its optimal strategy conditioned on the strategies of the other players. It is, as the name suggests, the best response that the player can give for a given strategy of the others. Let $x^{(u)}: \mathcal{X} \rightarrow \mathcal{X}$, defined as

$$
x^{(u)}(\mathbf{x})=\left(\arg \min _{\mathbf{z} \in \mathcal{X}_{u}} T_{u}\left(\mathbf{z}, \mathbf{x}_{-u}\right), \mathbf{x}_{-u}\right),
$$

be the best-response of user $u$ to the strategy $\mathbf{x}_{-u}$ of the other players. From the definition of $T_{u}$, it can be shown that for each $\mathbf{x} \in \mathcal{X}$, there is a unique $x^{(u)}(\mathbf{x})$. Given a point $\mathbf{x} \in \mathcal{X}$, the strategy profile $x^{(u)}(\mathbf{x})$ describes the strategies of all the players after the best response of user $u$.

Best-response dynamics then consists of players taking turns in some order to adapt their strategy based on the most recent known strategy of the others (without considering the effect on future play in the game).

Define a round to be a sequence of best-responses in which each player plays exactly once. Once an order is fixed in the first round, it is assumed to be the same in each subsequent round. The order in which the players best-respond in the first-round can be arbitrary. Let us fix this order to be $1,2, \ldots, K$.

Define $\hat{x}^{(1)}: \mathcal{X} \rightarrow \mathcal{X}$ as

$$
\hat{x}^{(1)}(\mathbf{x})=x^{(K)} \circ x^{(K-1)} \circ \ldots \circ x^{(1)}(\mathbf{x}),
$$


be the point reached from $\mathbf{x}$ after one round of play. One can recursively define

$$
\hat{x}^{(n)}(\mathbf{x})=\hat{x}^{(1)} \circ \hat{x}^{(n-1)}(\mathbf{x}),
$$

which is the point reached after $n$ rounds.

The best-response dynamics can then be defined as the sequence $\left\{\hat{x}^{(n)}\left(\mathbf{x}_{0}\right)\right\}_{n>1}$ cooresponding to the strategy of players after each round of best-response when $\mathbf{x}_{0}$ is the initial strategy. A NEP has the property that each player's strategy is a best-response to strategies of the other players. Therefore if $\mathbf{x}_{0}$ is a NEP then sequence will remain at $\mathbf{x}_{0}$.

The main question we seek to answer is: do the best-response dynamics for the routing game converge from any starting point? If they converge, then the converge to the Nash equilibrium point.

\section{The Non-linear Spectral Radius Approach}

A usual method to prove the convergence of iterates of an operator $\hat{x}^{(1)}: \mathcal{X} \rightarrow \mathcal{X}$ is to show that this operator is a contraction. For this, one needs to find a suitable norm, say $\|\cdot\|$, for which there exists a constant $c \in[0,1)$ such that

$$
\left\|\hat{x}^{(1)}(\mathbf{x})-\hat{x}^{(1)}(\mathbf{y})\right\| \leq c\|\mathbf{x}-\mathbf{y}\|,
$$

for every pair of points $\mathbf{x}$ and $\mathbf{y}$ in the set $\mathcal{X}$. The contraction condition says that the distance between iterates of the function starting from two different points decreases with each iteration. The constant $c$ depends on the norm, and for a continuously differentiable operator, it can be computed as $\sup _{\mathbf{x}}\left\|D \hat{x}^{(1)}(\mathbf{x})\right\|$, which is the supremum of the Jacobian over all points in the domain of the operator. It is then sufficient to find a norm in which the above condition is satisfied.

For the best-response function, it turns out that it is non-trivial to find such a norm, independently of the starting point, in which the distance decreases with every iteration. Instead, as will be seen later it will be sufficient to find a norm in which the distance decreases asymptotically and not with every iteration. This weaker condition can be formalized using the notion of the non-linear spectral radius described below.

For a function $f: \mathcal{X} \rightarrow \mathcal{X}$, define the set

$$
\mathcal{J}(f)=\{D f(x): f \text { is differentiable at } x\} .
$$

which is the set of Jacobian matrices of the function $f$ evaluated at all points at which $f$ is differentiable.

Definition 1 The non-linear spectral radius of a function $f: \mathcal{X} \rightarrow \mathcal{X}$ is defined as [25]:

$$
\bar{\rho}(f)=\lim \sup _{n \rightarrow \infty} \sup _{A_{i} \in \mathcal{J}(f)}\left\|\prod_{i=1}^{n} A_{i}\right\|^{1 / n} .
$$

The non-linear spectral radius of $f$ is related to the notion of joint spectral radius of a set $\mathcal{M}$ of matrices which is defined as:

$$
\hat{\rho}(\mathcal{M})=\lim \sup _{n \rightarrow \infty} \sup _{M_{i} \in \mathcal{M}}\left\|\prod_{i=1}^{n} M_{i}\right\|^{1 / n},
$$

and is independent of the induced matrix norm. It measures the worst case growth rate of a sequence of linear transformations that are taken from the set $\mathcal{M}$. It can been seen that the non-linear spectral radius of $f$ is in fact the joint spectral radius of the set of Jacobian matrices of $f, \mathcal{J}(f)$.

When there is only one matrix in $\mathcal{M}$, from Gelfand's formula it follows that the joint spectral radius is equal to the spectral radius of that matrix. For a set with several matrices, there is an equivalent result in terms of the generalized spectral radius of $\mathcal{M}$ which is defined as:

$$
\rho(\mathcal{M})=\limsup _{n \rightarrow \infty} \sup _{M_{i} \in \mathcal{M}} \rho\left(\prod_{i=1}^{n} M_{i}\right)^{\frac{1}{n}},
$$

where $\rho(A)$ is the spectral radius of the matrix $A$. If $\mathcal{M}$ is bounded then the generalized spectral radius and the joint spectral radius of $\mathcal{M}$ are equal 7 . 
Consider a linear dynamical system of the form

$$
x_{n+1}=A_{i(n)} x_{n},
$$

where the matrices $A_{i} \in \mathcal{M}$ can be chosen differently in each step. Such a system is called a switched linear system. When all the matrices are the same, one can determine the stability of such a system by checking whether the spectral radius of this matrix is less than 1 or not. In case of swithced linear systems, the same condition with the joint spectral radius in place of the spectral radius can be used to ascertain the stability of the system, see for example [34.

For non-linear operators, the following convergence criterion was stated in 25].

Theorem 1 ([25] Theorem 1) If $f: \mathcal{X} \rightarrow \mathcal{X}$ is Lipschitz-continuous and has a non-linear spectral radius smaller than 1 , then the iterates of $f$ are globally asymptotically stable. Moreover, the rate of exponential decay, $r$, satisfies $0<r \leq-\log (\bar{\rho}(f))$.

Thus, instead of requiring the best-response to be a contraction, one can show the convergence of the bestresponse dynamics by showing that:

1. $\hat{x}^{(1)}$ is Lipschitz-continuous; and

2. $\bar{\rho}\left(\hat{x}^{(1)}\right)<1$.

In the rest of this section, first we shall show a few properties of the best-response function, and then compute the structure of its Jacobian matrices, before arriving at our main result.

\subsection{Properties of the best-response function}

The purpose of this section is to establish various properties of best-response function, mainly related to its continuity and differentiability. Let us define

$$
\mathcal{S}_{u}(\mathbf{x})=\left\{j \in \mathcal{S}: x_{u, j}^{(u)}(\mathbf{x})>0\right\}
$$

as the set of links used by player $u$ in its best-response to the strategies $\mathbf{x}_{-u}$ of other players. We have the following result.

Theorem 2 The best-response function $x^{(u)}$ of player $u$ is Lipschitz-continuous on $\mathcal{X}$ with

$$
\left\|x^{(u)}(\mathbf{z})-x^{(u)}(\mathbf{w})\right\|_{1}<2\|\mathbf{z}-\mathbf{w}\|_{1}, \quad \forall \mathbf{z}, \mathbf{w} \in \mathcal{X} .
$$

Proof. See Appendix $\mathrm{A}$.

Corollary 1 Since the best-response over one round, $\hat{x}^{(1)}$, is a composition of best-responses of each of the players (cf. (6) ), it then follows that $\hat{x}^{(1)}$ is Lipschitz continuous.

Remark 2 The continuity of the best-response functions is a direct consequence of Berge's Theorem on the continuity of correspondances [6] (see also page 64 of [9]). However, Lipschitz continuity requires some more work than that.

Once the Lipschitz continuity of $\hat{x}^{(1)}$ has been established, it remains to be shown that its non-linear spectral radius is smaller than 1 . For this, we shall investigate the points at which the $\hat{x}^{(1)}$ is differentiable and compute the structure of its Jacobian.

We note that, according to Rademacher's theorem [15], a consequence of Theorem 2 is that the bestresponse function $x^{(u)}$ is Fréchet-differentiable almost everywhere in $\mathcal{X}$; that is, the points in $\mathcal{X}$ at which

$x^{(u)}$ is not differentiable form a set of Lebesgue measure zero. To compute the points at which the derivative is defined, we shall need the following definitions: 
- Let

$$
g_{i, j}(\mathbf{x})=\frac{\partial T_{i}}{\partial x_{i, j}}(\mathbf{x})=\pi_{j}\left(\phi\left(\frac{y_{j}}{r_{j}}\right)+\frac{x_{i, j}}{r_{j}} \phi^{\prime}\left(\frac{y_{j}}{r_{j}}\right)\right),
$$

where $y_{j}=\sum_{k} x_{k, j}$, be the marginal cost of player $i$ on link $j$ under strategy profile $\mathbf{x}$.

We say that link $j$ is marginally used by user $u$ at point $\mathbf{x}$ whenever the flow of user $u$ on that link is 0 although the marginal cost of that player on that link is minimum, that is

$$
x_{u, j}=0 \text { and } g_{u, j}(\mathbf{x})=\min _{k \in \mathcal{S}} g_{u, k}(\mathbf{x}) .
$$

- we say that the set $\mathcal{S}_{u}(\mathbf{x})$ is locally stable at point $\mathbf{x}$ if it does not change for an infinitesimal variation on the strategies of the other players, that is

$$
\exists \epsilon>0, \forall \mathbf{z} \in \mathcal{B}_{o}(\mathbf{x}, \epsilon), \mathcal{S}_{u}(\mathbf{x})=\mathcal{S}_{u}(\mathbf{z}) .
$$

From our assumptions on the function $\phi$, the continuity of the best-response functions imply that of the marginal costs $g_{i, j}$ defined in (14) under the best-response dynamics. In the following, we say that no link is marginally used by user $u$ in its best-response at point $\mathbf{x}$ if there is no link that is marginally used by user $u$ at point $x^{(u)}(\mathbf{x})$. The two notions introduced above are related through the following result.

Lemma 1 if there is no link that is marginally used by player $u$ in its best-response at point $\mathbf{x}$, then the set of links $\mathcal{S}_{u}(\mathbf{x})$ is locally stable at point $\mathbf{x}$.

Proof. See Appendix A.

Our first result regarding the differentiability of best-response functions is the following.

Proposition 1 The best-response function $x^{(u)}$ is differentiable at every point $\mathbf{x} \in \mathcal{X}$ such that no link is marginally used by player $u$ in its best-response at point $\mathbf{x}$.

Proof. See Appendix A.

\subsection{Structure of the Jacobian matrices}

The Jacobian matrix of $\hat{x}^{(1)}$ is the product of Jacobian matrices of best-responses of individual players. So, we shall start by computing the Jacobian of the best-response functions of individual players.

Consider a player $u$ and a point $\mathbf{x} \in \mathcal{X}$ at which $x^{(u)}$ is differentiable. The Jacobian matrix of this function is then the block matrix

$$
\mathrm{D} x^{(u)}(\mathbf{x})=\left(\begin{array}{ccc}
\frac{\partial x_{1}^{(u)}}{\partial \mathbf{x}_{1}}(\mathbf{x}) & \ldots & \frac{\partial x_{1}^{(u)}}{\partial \mathbf{x}_{K}}(\mathbf{x}) \\
\vdots & & \vdots \\
\frac{\partial x_{K}^{(u)}}{\partial \mathbf{x}_{1}}(\mathbf{x}) & \ldots & \frac{\partial x_{K}^{(u)}}{\partial \mathbf{x}_{K}}(\mathbf{x})
\end{array}\right),
$$

where the $(i, j)$-block $\frac{\partial x_{i}^{(u)}}{\partial \mathbf{x}_{j}}(\mathbf{x})$ measures the sensitivity of the strategy of player $i$ obtained after the best response of player $u$ with respect to a change in the strategy of player $j$.

The best-response of a player $u$ is sensitive only to the strategies of the other players $v \neq u$, and these sensitivities are reflected by the block matrices $\frac{\partial x_{u}^{(u)}}{\partial \mathbf{x}_{v}}$ which appear in the $u$ th row of the Jacobian matrix. Recalling that

$$
\frac{\partial x_{u}^{(u)}}{\partial \mathbf{x}_{v}}(\mathbf{x})=\left(\frac{\partial x_{u, i}^{(u)}}{\partial x_{v, j}}(\mathbf{x})\right)_{i \in \mathcal{S}, j \in \mathcal{S}},
$$

we shall distinguish between links $i \notin \mathcal{S}_{u}(\mathbf{x})$ and links $i^{\prime} \in \mathcal{S}_{u}(\mathbf{x})$. We assume in the following that the set $\mathcal{S}_{u}(\mathbf{x})$ is locally stable (cf. Section 3.1), and thus that it does not change for an infinitesimal variation on the strategy $\mathbf{x}_{v}$ of player $v \in \mathcal{C}$. 
Lemma 2 For all links $i \notin \mathcal{S}_{u}(\mathbf{x})$,

$$
\frac{\partial x_{u, i}^{(u)}}{\partial \mathbf{x}_{v}}(\mathbf{x})=0, \quad \forall v \in \mathcal{C}
$$

Proof. See Appendix B

For links $i \in \mathcal{S}_{u}(\mathbf{x})$, we have:

Lemma 3 There exist a vector $\boldsymbol{\theta} \in \mathbb{R}_{+}^{S}$ and a vector $\gamma \in \mathbb{R}_{+}^{S}$ satisfying $\gamma_{i}=0$ for all $i \notin \mathcal{S}_{u}(\mathbf{x})$ and $\sum_{i \in \mathcal{S}} \gamma_{i}=1$ such that

$$
\frac{\partial x_{u, i}^{(u)}}{\partial x_{v, k}}= \begin{cases}\theta_{i}\left(\gamma_{i}-1\right) & \text { if } k=i, \\ \theta_{k} \gamma_{i} & \text { otherwise, }\end{cases}
$$

for all players $v \neq u$ and all links $i \in \mathcal{S}_{u}(\mathbf{x})$ and $k \in \mathcal{S}$.

Proof. See Appendix B.

Remark 3 The vectors $\boldsymbol{\theta}$ and $\boldsymbol{\gamma}$ depend upon the strategy profile $\mathbf{x}$ and upon the player $u$ that updates its strategy. We have not made this dependence explicit in order to simplify the notation.

Further, the vector $\boldsymbol{\theta}$ has the following important property which will be helpful in establishing the desired inequality on the non-linear spectral radius of $\hat{x}^{(1)}$.

Lemma 4 There exists a constant $q<1$ such that

$$
\frac{1}{2} \leq \theta_{k} \leq q, \quad \forall k \in \mathcal{S}, \forall \mathbf{x} \in \mathcal{X}, \forall u \in \mathcal{C} .
$$

Proof. See Appendix B

The structure of the Jacobian matrices of the best-response functions is summarized in the following result.

Theorem 3 The Jacobian matrix of the best response function $x^{(u)}$ of player $u \in \mathcal{C}$ has the following form

$$
\mathrm{D} x^{(u)}(\mathbf{x})=\left(\begin{array}{ccccc}
I & \ldots & 0 & \ldots & 0 \\
\vdots & \ddots & & & \vdots \\
M_{u}(\mathbf{x}) & \ldots & 0 & \ldots & M_{u}(\mathbf{x}) \\
\vdots & & & \ddots & \vdots \\
0 & \ldots & 0 & \ldots & I
\end{array}\right)
$$

and $M_{u}(\mathbf{x})=\Psi(\Gamma B-I) \Theta$, where

- $B$ is the $S \times S$ matrix with 1 in every entry, i.e., $B=\mathbf{1}^{T} \mathbf{1}$,

- $\Gamma=\operatorname{diag}(\boldsymbol{\gamma})$ and $\Theta=\operatorname{diag}(\boldsymbol{\theta})$, the vectors $\boldsymbol{\gamma}$ and $\boldsymbol{\theta}$ being those defined in Lemma 3 ,

- $\Psi$ a positive diagonal matrix such that $\Psi_{i, i}=1$ if $i \in \mathcal{S}_{u}(\mathbf{x})$, and $\Psi_{i, i}=0$ otherwise.

Proof. The proof is broken down in three steps. Firstly, the $u$ th row follows directly from Lemma 3 Secondly, the strategies of all players except player $u$ do not change following the best response of player $i$. Therefore, for all $i \neq u$ and all $v \in \mathcal{C}$, we have

$$
\frac{\partial x_{i}^{(u)}}{\partial \mathbf{x}_{v}}(\mathbf{x})= \begin{cases}I & \text { if } v=i \\ 0 & \text { otherwise }\end{cases}
$$


This explains the appearance of the identity matrix on the diagonal and the 0 matrix in other columns of each row except the row corresponding to the player doing the best-response (that is, row $u$ ).

Finally, since the best response of player $u$ at point $\mathbf{x}$ is insensitive to her strategy at that point and depends only on the strategies of the other player, we can conclude that, for all $u \in \mathcal{C}$,

$$
\frac{\partial x_{u}^{(u)}}{\partial \mathbf{x}_{u}}(\mathbf{x})=0 .
$$

This explains why the diagonal block in the $u$ th row is 0 .

Corollary 2 The Jacobian matrix of $\hat{x}^{(1)}$ has the form

$$
\mathrm{D} \hat{x}^{(1)}(\mathbf{x})=\prod_{u=K}^{1} \mathrm{D} x^{(u)}(\mathbf{x}),
$$

where the index u goes down from $K$ to 1 .

\section{Convergence of best-response dynamics}

In this section, we shall first formulate a conjecture on the non-linear spectral radius of $\hat{x}^{(1)}$ on which the main result of this paper hinges. Then, this conjecture will be shown to be true for two particular cases : (a) two-player routing games; (b) $K$ player routing games with linear link cost function, $\phi$.

Conjecture 1 For a fixed $K$ and $S$, let $\hat{\mathcal{J}}$ be the set of matrices of the form given in Corollary 2 . Then, the joint spectral radius of $\hat{\mathcal{J}}$ is strictly less than 1 .

On the extensive numerical experiments that we conducted, the above conjecture was indeed true.

The main result of this paper is then:

Theorem 4 If Conjecture 1 is true, then the best-response dynamics (8) for the routing game (BR-i) converges to the unique Nash equilibrium of the game.

While we were unable to prove the conjecture, and hence the convergence of best-response dynamics, in its generality, we can show its validity for two non-trivial cases - the two player game, and the $K$ player game with linear link cost function, which we show below.

\subsection{Two-player routing game}

First, we shall prove a general result related to the Joint spectral radius of a certain class of matrices. The claimed result on the convergence of the best-response for the two-player game will then follow directly from that result.

Let $\mathcal{D}^{+}$be the set of positive diagonal matrices, and $\mathcal{G}$ be the set of diagonal matrices $\Gamma \in \mathcal{D}^{+}$whose diagonal entries satisfy in addition

$$
\sum_{i=1}^{S} \gamma_{i}=1
$$

For any natural number $k \geq 0$, the above two types of diagonal matrices are used to define the set $\mathcal{M}$ of $S \times S$ matrices as follows. $\mathcal{M}$ is the set of matrices $M$ that can be written as $M=(\Gamma B-I) \Theta$ for some matrices $\Gamma \in \mathcal{G}$ and $\Theta \in \mathcal{D}^{+}$. We also define $\mathcal{M}^{(k)}$ for $k \geq 0$ as the set of matrices that can be written as the product of $k$ matrices belonging to $\mathcal{M}$, where by convention $\mathcal{M}^{(0)}$ contains only the identity matrix.

For $q \in(0,1)$, we say that a matrix $M$ is in the set $\mathcal{M}_{q}$ if $M=(\Gamma B-I) \Theta \in \mathcal{M}$ and in addition $\|\Theta\|_{1} \leq q$. We similarly define $\mathcal{M}_{q}^{(k)}$ as the set of matrices that can be written as the product of $k$ matrices belonging to $\mathcal{M}_{q}$. We note that the set $\mathcal{M}_{q}$ is obviously bounded. 
According to Theorem 3 and Lemma 4 the Jacobian matrices of the best-response functions of players 1 and 2 have the following simple form:

$$
\mathrm{D} x^{(1)}(\mathbf{x})=\left(\begin{array}{cc}
0 & \Psi_{1} M_{1} \\
0 & I
\end{array}\right), \quad \text { and } \quad \mathrm{D} x^{(2)}(\mathbf{x})=\left(\begin{array}{cc}
I & 0 \\
\Psi_{2} M_{2} & 0
\end{array}\right),
$$

where $M_{1}, M_{2} \in \mathcal{M}_{q}$ for some $q<1$ and where $\Psi_{1}, \Psi_{2}$ are diagonal matrices with 0-1 entries on the diagonal. Using Corollary 2, the Jacobian of the best-response function over one round has the form

$$
\mathrm{D} \hat{\mathbf{x}}^{(1)}=\left(\begin{array}{cc}
0 & \Psi_{1} M_{1} \\
0 & \Psi_{2} M_{2} M_{1}
\end{array}\right)
$$

where $M_{1}, M_{2} \in \mathcal{M}_{q}$. It then follows that the structure of the product of $n$ Jacobian matrices has the following form.

Lemma 5 If $J_{1}, J_{2}, \ldots, J_{n} \in \mathcal{J}$, then

$$
\prod_{i=1}^{n} J_{i}=\left(\begin{array}{cc}
0 & \Psi_{1} X_{1}^{(2 n-1)} \\
0 & \Psi_{2} X_{2}^{(2 n)}
\end{array}\right)
$$

where $\Psi_{1}, \Psi_{2}$ are positive diagonal matrices with 0 -1 entries on the diagonal, $X_{1}^{(2 n-1)} \in \mathcal{M}_{q}^{(2 n-1)}$, and $X_{2}^{(2 n)} \in \mathcal{M}_{q}^{(2 n)}$.

Proof. See Appendix C]

Lemma 5 shows that the behaviour of a large product of Jacobian matrices is governed by the asymptotic behaviour of the matrices $X_{1}^{(n)}, X_{2}^{(n)}$. These matrices are themselves the product of matrices that belong to $\mathcal{M}_{q}$. This suggests to first characterize the asymptotic growth rate of products of matrices in $\mathcal{M}_{q}$. Our key result regarding this characteriztion is stated in theorem 5

Theorem 5 For any $k \geq 1$ and any matrix $M=\prod_{i=1}^{k}\left(\Gamma^{(i)} B-I\right) \Theta^{(i)}$ in $\mathcal{M}^{(k)}$, it holds that

$$
\rho(M) \leq \prod_{i=1}^{k} \theta_{\max }^{i},
$$

where $\theta_{\max }^{i}=\max _{1 \leq j \leq S} \theta_{j}^{(i)}$ for all $i=1, \ldots, k$.

Proof. See Appendix D,

The above theorem holds for any product of matrices belonging to $\mathcal{M}$. If we now restrict our attention to matrices belonging to $\mathcal{M}_{q}$, we obtain the following immediate corollary.

Corollary 3 For any product $M_{n} M_{n-1} \ldots M_{1}$ of matrices belonging to $\mathcal{M}_{q}$, we have $\rho\left(M_{n} M_{n-1} \ldots M_{1}\right) \leq$ $q^{n}$, implying that $\rho\left(\mathcal{M}_{q}\right) \leq q$.

Proof. See Appendix C,

We are now in position to prove that sequential best-response dynamics converges to the unique Nash equilibrium $\mathbf{x}^{*}$.

Theorem 6 For the two player routing game over parallel links, the sequential best-response dynamics converges to the unique Nash equilibrium for any initial point $\mathbf{x}_{0} \in \mathcal{X}$.

Proof. See Appendix C. 


\section{2 $K$ player games with linear link cost functions}

Consider $\phi(x)=x$, a delay function which is often used in congestion games to model delays in road networks.

From (55), it follows that $\theta_{k}=1 / 2$. Thus, the matrix $M_{u}$ in Theorem 3 is of the form $\frac{1}{2}(\Gamma B-I)$ for some $\Gamma \in \mathcal{G}$.

Theorem 7 For the $K$ player routing game over parallel links and linear delay function, the sequential best-response dynamics converges to the unique Nash equilibrium for any initial point $\mathbf{x}_{0} \in \mathcal{X}$.

Proof. See Appendix E

\section{References}

[1] A. Akella, S. Seshan, R. Karp, S. Shenker, and C. Papadimitriou. Selfish behavior and stability of the internet: a game-theoretic analysis of tcp. In Proceedings of the 2002 conference on Applications, technologies, architectures, and protocols for computer communications, Pittsburgh, Pennsylvania, USA, August 19-23 2002.

[2] E. Altman, T. Basar, T. Jiménez, and N. Shimkin. Routing into two parallel links: Game-theoretic distributed algorithms. Journal of Parallel and Distributed Computing, 61(9):1367-1381, September 2001.

[3] E. Altman, T. Boulogne, R. E. Azouzi, T. Jimenez, and L. Wynter. A survey on networking games in telecommunications. Computers and Operations Research, 33(2):286-311, February 2006.

[4] J. Anselmi and B. Gaujal. Optimal routing in parallel, non-observable queues and the price of anarchy revisited. In 22nd International Teletraffic Congress (ITC), Amsterdam, 2010.

[5] U. Ayesta, O. Brun, and B. J. Prabhu. Price of anarchy in non-cooperative load balancing games. Performance Evaluation, 68:1312-1332, 2011.

[6] C. Berge. Espaces topologiques et fonctions multivoques. Dunod, Paris, [translation: topological spaces. new york: macmillan, 1963.1] edition, 1959.

[7] M. A. Berger and Y. Wang. Bounded semigroups of matrices. Linear Algebra and its Applications, (166):21-27, 1992.

[8] N. Berger, M. Feldman, O. Neiman, and M. Rosenthal. Dynamic inefficiency: Anarchy without stability. In 4th Symposium on Algorithmic Game Theory, October 2011.

[9] K. C. Border. Fixed point theorems with applications to economics and game theory. Cambridge University Press, 1985.

[10] O. Brun and B. Prabhu. Worst-case analysis of non-cooperative load balancing. Technical Report no 12590, LAAS-CNRS, 2012.

[11] D. E. Charilas and A. D. Panagopoulos. A survey on game theory applications in wireless networks. Computer Networks, 54(18):3421-3430, December 2010.

[12] H. L. Chen, J. Marden, and A. Wierman. The effect of local scheduling in load balancing designs. In Proceedings of IEEE Infocom, 2009.

[13] R. Cominetti, J. R. Correa, and N. E. Stier-Moses. The impact of oligopolistic competition in networks. Operations Research, Published online in Articles in Advance, DOI: 10.1287/opre.1080.0653, June 2009.

[14] A. Czumaj, P. Krysta, and B. Vocking. Selfish traffic allocation for server farms. In Proceedings of STOC, 2002. 
[15] L. C. Evans and R. F. Gariepy. Measure theory and fine Properties of Functions. Studies in Advanced Mathematics. CRC Press, Boca Raton, Florida, 1992.

[16] E. Even-Dar, A. Kesselman, and Y. Mansour. Convergence time to nash equilibria. In Proceedings of the 30th international conference on Automata, languages and programming, ICALP'03, pages 502-513, Berlin, Heidelberg, 2003. Springer-Verlag.

[17] A. Fabrikant, C. Papadimitriou, and K. Talwar. The complexity of pure nash equilibria. In A. Press, editor, Proceedings of the 36th annual ACM Symposium on Theory of Computing (STOC'04), pages 604-612, 2004.

[18] R. Garg, A. Kamra, and V. Khurana. A game-theoretic approach towards congestion control in communication networks. ACM SIGCOMM Computer Communication Review, 32(3):47-61, July 2002.

[19] M. Goemans, V. Mirrokni, and A. Vetta. Sink equilibria and convergence. In Proceedings of the 46th annual IEEE Symposium on Foundations of Computer Science (FOCS'05), pages 142-154, 2005.

[20] M. Haviv and T. Roughgarden. The price of anarchy in an exponential multi-server. Operations Research Letters, 35:421-426, 2007.

[21] I. Keidar, R. Melamed, and A. Orda. Equicast: Scalable multicast with selfish users. Computer Networks, 53(13), August 2009.

[22] E. Koutsoupias and C. H. Papadimitriou. Worst-case equilibria. In STACS 1999, 1999.

[23] A. Leshem and E. Zehavi. Cooperative game theory and the gaussian interference channel. IEEE Journal on Selected Areas in Communications, 26:1078-1088, 2008.

[24] L. López, G. del Rey Almansa, S. Paquelet, and A. Fernández. A mathematical model for the tcp tragedy of the commons. Theoretical Computer Science, 343(1-2):4-26, October 2005.

[25] K. Mak, J. Peng, Z. Xu, and K. Yiu. A new stability criterion for discrete-time neural networks: Nonlinear spectral radius. Chaos, Solitons and Fractals, 31(2):424 - 436, 2007.

[26] R. Menon, A. MacKenzie, J. Hicks, R. Buehrer, and J. Reed. A game-theoretic framework for interference avoidance. IEEE Transactions on Communications, 57(4):1087-1098, 2009.

[27] G. Mertzios. Fast convergence of routing games with splittable flows. In Proceedings of the 2nd International Conference on Theoretical and Mathematical Foundations of Computer Science (TMFCS), pages pp. 28-33, Orlando, FL, USA, July 2009.

[28] N. Nisan, T. Roughgarden, E. Tardos, and V. Vazirani. Algorithmic Game Theory. Cambridge University Press, New York, NY, USA, 2007.

[29] A. Orda, R. Rom, and N. Shimkin. Competitive routing in multi-user communication networks. IEEE/ACM Transactions on Networking, 1:510-521, October 1993.

[30] T. Roughgarden. The price of anarchy is independent of the network topology. J. Comput. Syst. Sci., 67(2):341-364, 2003.

[31] T. Roughgarden. Intrinsic robustness of the price of anarchy. In STOC '09, 2009.

[32] T. Roughgarden and E. Tardos. How bad is selfish routing? J. ACM, 49(2), March 2002.

[33] S. J. Shenker. Making greed work in networks: a game-theoretic analysis of switch service disciplines. IEEE/ACM Transactions on Networking (TON), 3(6):819-831, December 1995.

[34] J. Theys. Joint Spectral Radius: theory and approximations. PhD thesis, Center for Systems Engineering and Applied Mechanics, Université Catholique de Louvain, May 2005.

[35] T. Wu and D. Starobinski. On the price of anarchy in unbounded delay networks. In GameNets '06: Proceeding from the 2006 workshop on Game theory for communications and networks, page 13, New York, NY, USA, 2006. ACM. 


\section{A Proofs of results in Section 3.1}

Proof of Theorem 2. Consider two points $\mathbf{z}$ and $\mathbf{w}$ in $\mathcal{X}$. Let the vectors $\mathbf{a}, \mathbf{b} \in \mathbb{R}_{+}^{S}$ be such that $a_{j}=\sum_{i \neq u} z_{i, j}$ and $b_{j}=\sum_{i \neq u} w_{i, j}$ for all $j \in \mathcal{S}$. In other words, $a_{j}$ and $b_{j}$ are the total traffic sent on link $j$ by users other than $u$ in configurations $\mathbf{z}$ and $\mathbf{w}$, respectively. To simplify notations, we denote by $x_{u, j}^{z}$ and $x_{u, j}^{w}$ the traffic sent on link $j$ by player $u$ after his best-response at points $\mathbf{z}$ and $\mathbf{w}$, respectively, that is $x_{u, j}^{z}=x_{u, j}^{(u)}(\mathbf{z})$ and $x_{u, j}^{w}=x_{u, j}^{(u)}(\mathbf{w})$. For the purpose of the proof, we also define

$$
f_{j}(x, y)=\pi_{j}\left(\phi\left(\frac{x+y}{r_{j}}\right)+\frac{x}{r_{j}} \phi^{\prime}\left(\frac{x+y}{r_{j}}\right)\right),
$$

for all links $j \in \mathcal{S}$. Then the marginal costs of player $u$ on link $j$ after the best-response of that player at points $\mathbf{z}$ and $\mathbf{w}$ can be written as $g_{u, j}\left(x^{(u)}(\mathbf{z})\right)=f_{j}\left(x_{u, j}^{z}, a_{j}\right)$ and $g_{u, j}\left(x^{(u)}(\mathbf{w})\right)=f_{j}\left(x_{u, j}^{w}, b_{j}\right)$. From the KKT conditions, there exist $\mu_{z}$ and $\mu_{w}$ such that $f_{j}\left(x_{u, j}^{z}, a_{j}\right) \geq \mu_{z}$, with equality if $j \in \mathcal{S}_{u}(\mathbf{z})$, and $f_{j}\left(x_{u, j}^{w}, b_{j}\right) \geq \mu_{w}$, with equality if $j \in \mathcal{S}_{u}(\mathbf{w})$. Without loss of generality, we assume that $\mu_{z} \geq \mu_{w}$. As a consequence, we have

$$
f_{j}\left(x_{u, j}^{z}, a_{j}\right) \geq f_{j}\left(x_{u, j}^{w}, b_{j}\right), \quad \forall j \in \mathcal{S}_{u}(\mathbf{w}) .
$$

Consider now the sets

$$
\mathcal{S}^{-}=\left\{j \in \mathcal{S}: x_{u, j}^{z}<x_{u, j}^{w}\right\}
$$

and

$$
\mathcal{S}^{+}=\left\{j \in \mathcal{S}: x_{u, j}^{z} \geq x_{u, j}^{w}\right\} .
$$

Assume first that $\mathcal{S}^{-}=\emptyset$. Then $x_{u, j}^{z} \geq x_{u, j}^{w}$ for all $j \in \mathcal{S}$. However, since

$$
\sum_{j \in \mathcal{S}} x_{u, j}^{z}=\sum_{j \in \mathcal{S}} x_{u, j}^{w}=\lambda_{u}
$$

this implies that $x_{u, j}^{z}=x_{u, j}^{w}$ for all $j \in \mathcal{S}$. It yields

$$
\sum_{j \in \mathcal{S}}\left|x_{u, j}^{z}-x_{u, j}^{w}\right|=0
$$

Assume now that $\mathcal{S}^{-} \neq \emptyset$. Since $\mathcal{S}=\mathcal{S}^{-} \cup \mathcal{S}^{+}$, we obtain from that

$$
\sum_{j \in \mathcal{S}^{+}}\left|x_{u, j}^{z}-x_{u, j}^{w}\right|=-\sum_{j \in \mathcal{S}^{-}}\left|x_{u, j}^{z}-x_{u, j}^{w}\right|,
$$

which leads to

$$
\sum_{j \in \mathcal{S}}\left|x_{u, j}^{z}-x_{u, j}^{w}\right|=2 \sum_{j \in \mathcal{S}^{-}}\left|x_{u, j}^{z}-x_{u, j}^{w}\right| .
$$

For $j \in \mathcal{S}^{-}$, we have by definiton $0 \leq x_{u, j}^{z}<x_{u, j}^{w}$, and hence $j \in \mathcal{S}_{u}(\mathbf{w})$. Thus, $\mathcal{S}^{-} \subset \mathcal{S}_{u}(\mathbf{w})$. With (27), it yields $f_{j}\left(x_{u, j}^{z}, a_{j}\right) \geq f_{j}\left(x_{u, j}^{w}, b_{j}\right)$, and thus

$$
\phi\left(\frac{x_{u, j}^{z}+a_{j}}{r_{j}}\right)+\frac{x_{u, j}^{z}}{r_{j}} \phi^{\prime}\left(\frac{x_{u, j}^{z}+a_{j}}{r_{j}}\right) \geq \phi\left(\frac{x_{u, j}^{w}+b_{j}}{r_{j}}\right)+\frac{x_{u, j}^{w}}{r_{j}} \phi^{\prime}\left(\frac{x_{u, j}^{w}+b_{j}}{r_{j}}\right),
$$

for all $j \in \mathcal{S}^{-}$. However, since for $j \in \mathcal{S}^{-}$we have $x_{u, j}^{z}<x_{u, j}^{w}$ and since $\phi$ and $\phi^{\prime}$ are strictly increasing, this implies that $x_{u, j}^{z}+a_{j}>x_{u, j}^{w}+b_{j}$, from which we deduce that

$$
0<x_{u, j}^{w}-x_{u, j}^{z}<a_{j}-b_{j} \quad \forall j \in \mathcal{S}^{-} .
$$

It yields

$$
\sum_{j \in \mathcal{S}^{-}}\left|x_{u, j}^{z}-x_{u, j}^{w}\right|<\sum_{j \in \mathcal{S}^{-}}\left|a_{j}-b_{j}\right|
$$


With (33), we thus obtain

$$
\sum_{j \in \mathcal{S}}\left|x_{u, j}^{z}-x_{u, j}^{w}\right|<2 \sum_{j \in \mathcal{S}^{-}}\left|a_{j}-b_{j}\right| \leq 2 \sum_{j \in \mathcal{S}}\left|a_{j}-b_{j}\right|
$$

From (31) and (36), we obtain that, whether $\mathcal{S}^{-}$be empty or not, we have

$$
\begin{aligned}
\sum_{j \in \mathcal{S}}\left|x_{u, j}^{z}-x_{u, j}^{w}\right| & <2 \sum_{j \in \mathcal{S}}\left|\sum_{i \neq u} z_{i, j}-\sum_{i \neq u} w_{i, j}\right| \\
& <2 \sum_{j \in \mathcal{S}} \sum_{i \neq u}\left|z_{i, j}-w_{i, j}\right| \\
& <2 \sum_{i \in \mathcal{C}} \sum_{j \in \mathcal{S}}\left|z_{i, j}-w_{i, j}\right|
\end{aligned}
$$

Since $x_{i, j}^{(u)}(\mathbf{z})=x_{i, j}^{(u)}(\mathbf{w})$ for all $j \in \mathcal{S}$ and all $i \neq u$, we also have

$$
\sum_{i \neq u} \sum_{j \in \mathcal{S}}\left|x_{i, j}^{(u)}(\mathbf{z})-x_{i, j}^{(u)}(\mathbf{w})\right|=0
$$

Finally, from (37) and (38), we conclude that

$$
\sum_{i \in \mathcal{C}} \sum_{j \in \mathcal{S}}\left|x_{i, j}^{(u)}(\mathbf{z})-x_{i, j}^{(u)}(\mathbf{w})\right|<2 \sum_{i \in \mathcal{C}} \sum_{j \in \mathcal{S}}\left|z_{i, j}-w_{i, j}\right|,
$$

that is,

$$
\left\|x^{(u)}(\mathbf{z})-x^{(u)}(\mathbf{w})\right\|_{1}<2\|\mathbf{z}-\mathbf{w}\|_{1},
$$

as claimed.

Proof of Lemma 1. Let $\Omega_{u}$ be the set of points $\mathbf{x} \in \mathcal{X}$ where $\mathcal{S}_{u}(\mathbf{x})$ is locally stable. Let us define

$$
f_{j}(x, y)=\pi_{j}\left(\phi\left(\frac{x+y}{r_{j}}\right)+\frac{x}{r_{j}} \phi^{\prime}\left(\frac{x+y}{r_{j}}\right)\right),
$$

for all links $j \in \mathcal{S}$. Note that $f_{j}(x, y)$ is continuous and strictly increasing in both $x$ and $y$. Then the marginal cost of player $u$ on link $j$ after the best-response of that player can be written as $g_{u, j}\left(x^{(u)}(\mathbf{x})\right)=$ $f_{j}\left(x_{u, j}^{(u)}(\mathbf{x}), \sum_{k \neq u} x_{k, j}\right)$. From the KKT conditions, the function $\mu: \mathcal{X}_{-u} \rightarrow \mathbb{R}$ defined by

$$
\mu\left(\mathbf{x}_{-u}\right)=\min _{j \in \mathcal{S}} g_{u, j}\left(x^{(u)}(\mathbf{x})\right)
$$

is such that

$$
j \in \mathcal{S}_{u}(\mathbf{x}) \Longleftrightarrow f_{j}\left(0, \sum_{k \neq u} x_{k, j}\right)<\mu\left(\mathbf{x}_{-u}\right) .
$$

Note that the continuity of the best-response function $x^{(u)}$ on $\mathcal{X}$ (cf. Theorem 2) implies that of the marginal costs, and therefore the continuity of $\mu$ on $\mathcal{X}_{-u}$.

Let $\mathbf{x}$ be a point such that no link is marginally used by player $u$ in its best-response at point $\mathbf{x}$. Let us first consider $j \in \mathcal{S}_{u}(\mathbf{x})$. From (41), there exists $\delta>0$ such that $f_{j}\left(0, \sum_{k \neq u} x_{k, j}\right) \leq \mu\left(\mathbf{x}_{-u}\right)-\delta$. Since $f_{j}(x, y)$ is continuous in $y$ and $\mu\left(\mathbf{x}_{-u}\right)$ is continuous on $\mathcal{X}_{-u}$, there exists $\epsilon_{1}>0$ such that, for all $\mathbf{z} \in \mathcal{B}_{o}\left(\mathbf{x}, \epsilon_{1}\right)$,

$$
f_{j}\left(0, \sum_{k \neq u} z_{k, j}\right)<f_{j}\left(0, \sum_{k \neq u} x_{k, j}\right)+\frac{\delta}{2}<\mu\left(\mathbf{x}_{-u}\right)-\frac{\delta}{2},
$$

and $\mu\left(\mathbf{z}_{-u}\right)>\mu\left(\mathbf{x}_{-u}\right)-\frac{\delta}{2}$. It yields 


$$
f_{j}\left(0, \sum_{k \neq u} z_{k, j}\right)<\mu\left(\mathbf{x}_{-u}\right)-\frac{\delta}{2}<\mu\left(\mathbf{z}_{-u}\right), \quad \forall \mathbf{z} \in \mathcal{B}_{o}\left(\mathbf{x}, \epsilon_{1}\right),
$$

and thus, according to (41), we have $j \in \mathcal{S}_{u}(\mathbf{z})$ for all $\mathbf{z} \in \mathcal{B}_{o}\left(\mathbf{x}, \epsilon_{1}\right)$ if $j \in \mathcal{S}_{u}(\mathbf{x})$. As a consequence, if $\mathcal{S}_{u}(\mathbf{x})=\mathcal{S}$, then $\mathcal{S}_{u}(\mathbf{z})=\mathcal{S}$ for all $\mathbf{z}$ sufficiently close to $\mathbf{x}$, and thus $\mathbf{x} \in \Omega_{u}$.

Otherwise we can find $j \in \mathcal{S} \backslash \mathcal{S}_{u}(\mathbf{x})$. Since no link is marginally used by player $u$ in its best-response at point $\mathbf{x}$, there exists $\beta>0$ such that

$$
f_{j}\left(0, \sum_{k \neq u} x_{k, j}\right) \geq \mu\left(\mathbf{x}_{-u}\right)+\beta, \quad \forall j \in \mathcal{S} \backslash \mathcal{S}_{u}(\mathbf{x}) .
$$

Proceeding as above, we can show that there exists $\epsilon_{2}>0$ such that, for all $\mathbf{z} \in \mathcal{B}_{o}\left(\mathbf{x}, \epsilon_{2}\right), \mu\left(\mathbf{z}_{-u}\right)<$ $\mu\left(\mathbf{x}_{-u}\right)+\frac{\beta}{2}$ and

$$
f_{j}\left(0, \sum_{k \neq u} z_{k, j}\right)>f_{j}\left(0, \sum_{k \neq u} x_{k, j}\right)-\frac{\beta}{2}>\mu\left(\mathbf{x}_{-u}\right)+\frac{\beta}{2},
$$

from which we conclude that $f_{j}\left(0, \sum_{k \neq u} z_{k, j}\right)>\mu\left(\mathbf{z}_{-u}\right)$, for all $\mathbf{z} \in \mathcal{B}_{o}\left(\mathbf{x}, \epsilon_{2}\right)$. This implies that if $j \notin \mathcal{S}_{u}(\mathbf{x})$, then $j \notin \mathcal{S}_{u}(\mathbf{z})$ for all $\mathbf{z} \in \mathcal{B}_{o}\left(\mathbf{x}, \epsilon_{2}\right)$.

Choosing $\epsilon=\min \left(\epsilon_{1}, \epsilon_{2}\right)$, we thus conclude that for all $\mathbf{z} \in \mathcal{B}_{o}(\mathbf{x}, \epsilon), \mathcal{S}_{u}(\mathbf{x}) \subset \mathcal{S}_{u}(\mathbf{z})$ and $\mathcal{S} \backslash \mathcal{S}_{u}(\mathbf{x}) \subset$ $\mathcal{S} \backslash \mathcal{S}_{u}(\mathbf{z})$, which is equivalent to $\mathcal{S}_{u}(\mathbf{x})=\mathcal{S}_{u}(\mathbf{z})$. This shows that if no link is marginally used by player $u$ in its best-response at point $\mathbf{x}$, then $\mathcal{S}_{u}(\mathbf{x})$ is locally stable.

Proof of Proposition 1. From Lemma 1, we know that if $\mathrm{x}$ is such that no link is marginally used by user $u$ in its best-response at point $\mathbf{x}$, then the set of links $\mathcal{S}_{u}(\mathbf{x})$ is locally stable at $\mathbf{x}$. As shown in Theorem 3. this condition is sufficient to compute the partial derivatives of $x^{(u)}$ at point $\mathbf{x}$. It can be seen from (21) and (22) that the partial derivatives $\frac{\partial x_{i}^{(u)}}{\partial \mathbf{x}_{v}}(\mathbf{x}), i \neq u, v \in \mathcal{C}$, and $\frac{\partial x_{u}^{(u)}}{\partial \mathbf{x}_{u}}(\mathbf{x})$ are contiuous at $\mathbf{x}$. According to Lemma 2, the continuity of the partial derivatives $\frac{\partial x_{u, i}^{(u)}}{\partial \mathbf{x}_{v}}(\mathbf{x})$ at $\mathbf{x}$ for $i \notin \mathcal{S}_{u}(\mathbf{x})$ follows from the local stability of $\mathcal{S}_{u}(\mathbf{x})$ at $\mathbf{x}$. Finally, a closed-form formula is given in (19) for the partial derivatives $\frac{\partial x_{u, i}^{(u)}}{\partial x_{v, k}}$ for $v \neq u$ and for $i \in \mathcal{S}_{u}(\mathbf{x}), k \in \mathcal{S}$. In view of equations (50)-(54), the continuity of these partial derivatives follows from our assumptions on $\phi$ and from the continuity of $x^{(u)}$ at $\mathbf{x}$. Thus, all partial derivatives of $x^{(u)}$ exist and are continuous at $\mathbf{x}$, and therefore $x^{(u)}$ is continuously differentiable at $\mathbf{x}$.

\section{B Proofs of results in Section 3.2}

Proof of Lemma 2. The proof follows from the assumption that $\mathcal{S}_{u}(\mathbf{x})$ is locally stable at $\mathbf{x}$. We have $x_{u, i}^{(u)}(\mathbf{x}+h \mathbf{y})=x_{u, i}^{(u)}(\mathbf{x})=0$ for any vector $\mathbf{y}$ and $h>0$ sufficiently small. This implies that the directional derivatives of $x_{u, i}^{(u)}$, and thus its partial derivatives, are 0 .

Proof of Lemma 3. The proof is based on two observations: (i) at a best-reponse strategy, the change in marginal cost of player $u$ due to a change in the strategy of player $v$ is the same in every link that is used at the best-response strategy; and (ii) the total flow is conserved for player $u$ irrespective of the change in the strategy of player 1.

Recall that

$$
g_{u, i}\left(x^{(u)}(\mathbf{x})\right):=\frac{\partial T_{u}}{\partial x_{u, i}}\left(x^{(u)}(\mathbf{x})\right) .
$$

is the marginal cost of player $u$ at link $i$ under strategy profile $x^{(u)}(\mathbf{x})$, i.e., after the best-response of player $u$.

For $i \in \mathcal{S}_{u}(\mathbf{x})$, from the $K K T$ conditions, the best-response strategy of player $u, \mathbf{x}_{u}^{(u)}$, is such that the marginal cost is the same in all the links that receive a positive traffic at this strategy. That is,

$$
g_{u, i}\left(x^{(u)}(\mathbf{x})\right)=\mu\left(\mathbf{x}_{-u}\right) \quad \forall i \in \mathcal{S}_{u}(\mathbf{x}),
$$


where the constant $\mu$ depends upon the strategies of the players but not on the index of the link. The local stability of $\mathcal{S}_{u}(\mathbf{x})$ implies that the set of links used by user $u$ does not change for an infinitesimal variation on the strategies of the other players. This leads to our first observation which is that the change in the marginal cost of player $u$ at its best-response strategy due to the change in the strategy of player $v \neq u$ at link $k$ is the same at all links that receive a positive flow of player $u$. Thus,

$$
\frac{\partial g_{u, i}}{\partial x_{v, k}}\left(x^{(u)}(\mathbf{x})\right)=\mu_{2}, \quad \forall i \in \mathcal{S}_{u}(\mathbf{x}),
$$

where $\mu_{2}$ depends upon the strategies of the players. We have not made this dependence explicit in order to simplify the notation.

For a function of the form $h(f(x), x)$, its derivative with respect to $x$ is given by

$$
\frac{d h(f(x), x)}{d x}=\frac{d h(f, x)}{d f} \frac{d f}{d x}+\frac{d h(f, x)}{d x}
$$

where in the first term on the RHS, $h$ is treated to as a function of $f$ only, whereas in the second term it is treated as a function of $x$ only.

Since $x_{u, i}^{(u)}$ is a function of $x_{v, k}$, we can use the above formula to rewrite (44) as

$$
\frac{d g_{u, i}}{d x_{u, i}} \frac{\partial x_{u, i}^{(u)}}{\partial x_{v, k}}+\frac{d g_{u, i}}{d x_{v, k}}=\mu_{2}, \quad \forall i \in \mathcal{S}_{u}(\mathbf{x}),
$$

where the partial derivates are replaced by full derivates in order to indicate that the function is differentied in one variable while treating the other as constant.

The particular form of the cost function given in problem $(\overline{(B R-i})$ ) permits a simplification of the LHS of the above equation by noting that the marginal cost in a link depends only on the traffic that is routed to that link. Thus,

$$
\frac{d g_{u, i}}{d x_{u, i}} \frac{\partial x_{u, i}^{(u)}}{\partial x_{v, k}}+\delta_{k}(i) \frac{d g_{u, i}}{d x_{v, k}}=\mu_{2}, \quad \forall i \in \mathcal{S}_{u}(\mathbf{x})
$$

where $\delta_{k}(i)$ is unity if $i=k$, and is zero otherwise.

The value of $\mu_{2}$ can be computed using the second observation that the total flow of player $u$ is conserved irrespective of the strategy of player $v$. That is,

$$
\sum_{i \in \mathcal{S}_{u}(\mathbf{x})} \frac{\partial x_{u, i}^{(u)}}{\partial x_{v, k}}=0
$$

We thus obtain

$$
\begin{aligned}
\mu_{2} & =\left(\sum_{l \in \mathcal{S}_{u}(\mathbf{x})} \delta_{k}(l) \frac{d g_{u, l}}{d x_{v, k}}\left(\frac{d g_{u, l}}{d x_{u, l}}\right)^{-1}\right)\left(\sum_{l \in \mathcal{S}_{u}(\mathbf{x})}\left(\frac{d g_{u, l}}{d x_{u, l}}\right)^{-1}\right)^{-1} \\
& =\left(\frac{d g_{u, k}}{d x_{v, k}}\left(\frac{d g_{u, k}}{d x_{u, k}}\right)^{-1}\right)\left(\sum_{l \in \mathcal{S}_{u}(\mathbf{x})}\left(\frac{d g_{u, l}}{d x_{u, l}}\right)^{-1}\right)^{-1}
\end{aligned}
$$

and

$$
\frac{\partial x_{u, i}^{(u)}}{\partial x_{v, k}}=\theta_{k}\left(\gamma_{i}-\delta_{k}(i)\right), \quad \forall i \in \mathcal{S}_{u}(\mathbf{x})
$$

where

$$
\theta_{k}=\frac{d g_{u, k}}{d x_{v, k}}\left(\frac{d g_{u, k}}{d x_{u, k}}\right)^{-1}
$$


and

$$
\gamma_{i}=\left(\sum_{l \in \mathcal{S}_{u}(\mathbf{x})}\left(\frac{d g_{u, l}}{d x_{u, l}}\right)^{-1}\right)^{-1}\left(\frac{d g_{u, i}}{d x_{u, i}}\right)^{-1} .
$$

We will now show that $0<\theta_{k}<1$ and $0<\gamma_{i}<1$. We have

$$
g_{u, k}=\pi_{k}\left(\phi\left(\rho_{k}\right)+\frac{x_{u, k}}{r_{k}} \phi^{\prime}\left(\rho_{k}\right)\right) .
$$

Thus, since $\phi$ is an increasing and convex function,

$$
\frac{d g_{u, k}}{d x_{v, k}}=\frac{\pi_{k}}{r_{k}}\left(\phi^{\prime}\left(\rho_{k}\right)+\frac{x_{u, k}}{r_{k}} \phi^{\prime \prime}\left(\rho_{k}\right)\right)>0,
$$

independently of the player $v \neq u$, and

$$
\frac{d g_{u, k}}{d x_{u, k}}=\frac{\pi_{k}}{r_{k}}\left(2 \phi^{\prime}\left(\rho_{k}\right)+\frac{x_{u, k}}{r_{k}} \phi^{\prime \prime}\left(\rho_{k}\right)\right)>0
$$

Thus, from (50), $\theta_{k}>0$ and

$$
\theta_{k}=\frac{\phi^{\prime}\left(\rho_{k}\right)+\frac{x_{u, k}}{r_{k}} \phi^{\prime \prime}\left(\rho_{k}\right)}{2 \phi^{\prime}\left(\rho_{k}\right)+\frac{x_{u, k}}{r_{k}} \phi^{\prime \prime}\left(\rho_{k}\right)}<1 .
$$

We thus obtain that $\theta_{k}$ is independant of $v$ and that $0<\theta_{k}<1$. Similarly, we note that $\gamma_{i}$ is positive and smaller than unity due to the fact that $\frac{d g_{0, l}}{d x_{0, k}^{(1)}}$ is postive for all $l$. To conclude the proof, we note that $\sum_{i \in \mathcal{S}_{u}(\mathbf{x})} \gamma_{i}=1$ from the definition of the vector $\gamma$ in (51). Thus, letting $\gamma_{i}=0$ for $i \notin \mathcal{S}_{u}(\mathbf{x})$, we obtain $\sum_{i \in \mathcal{S}} \gamma_{i}=1$.

In order to prove Lemma 4, we need the following result.

Lemma 6 There exists a strictly positive constant $\rho_{\max }<1$, independant of $u$ and $\mathbf{x}$, such that the utilization rate of each and every link $j \in \mathcal{S}_{u}(\mathbf{x})$ is upper bounded by $\rho_{\max }$ after the best-response of user $u$ at point $\mathbf{x}$, that is,

$$
\rho_{j}^{(u)}(\mathbf{x}) \leq \rho_{\max }, \quad \forall j \in \mathcal{S}_{u}(\mathbf{x}), \forall \mathbf{x} \in \mathcal{X}, \forall u \in \mathcal{C},
$$

where $\rho_{j}^{(u)}(\mathbf{x})=\frac{1}{r_{j}} \sum_{i \in \mathcal{C}} x_{i, j}^{(u)}(\mathbf{x})$.

Proof of Lemma 6. Observe that $\mathbf{x} \in \mathcal{X}$ implies that $\sum_{k \neq u} x_{k, j}<r_{j}$ for all links $j$, and thus that the optimization problem for player $u$ is well-defined. Let $\mathbf{z}=x^{(u)}(\mathbf{x})$ be the point reached after the best response of player $u$. To simplify notations, we let $\rho_{j}=\rho_{j}^{(u)}(\mathbf{x})$. From the $K K T$ conditions, there exists $\mu_{u}\left(\mathbf{x}_{-u}\right)$ such that

$$
\begin{aligned}
\pi_{j}\left[\phi\left(\rho_{j}\right)+\frac{z_{u, j}}{r_{j}} \phi^{\prime}\left(\rho_{j}\right)\right] & =\mu_{u}\left(\mathbf{x}_{-u}\right), \forall j \in \mathcal{S}_{u}(\mathbf{x}) \\
\pi_{j} \phi\left(\rho_{j}\right) & \geq \mu_{u}\left(\mathbf{x}_{-u}\right), \forall j \notin \mathcal{S}_{u}(\mathbf{x})
\end{aligned}
$$

Since $0 \leq z_{u, j} / r_{j} \leq \rho_{j}, \forall j \in \mathcal{S}_{u}(\mathbf{x})$, (57) leads to the inequalities

$$
\begin{aligned}
\pi_{j} \phi\left(\rho_{j}\right) & \leq \mu_{u}\left(\mathbf{x}_{-u}\right), \\
\mu_{u}\left(\mathbf{x}_{-u}\right) & \leq \pi_{j}\left(\rho_{j} \phi^{\prime}\left(\rho_{j}\right)+\phi\left(\rho_{j}\right)\right) .
\end{aligned}
$$

Moreover, $\rho_{j}$ and $\phi^{\prime}\left(\rho_{j}\right)$ are non-negative. Thus, (58) leads to

$$
\mu_{u}\left(\mathbf{x}_{-u}\right) \leq \pi_{j}\left(\rho_{j} \phi^{\prime}\left(\rho_{j}\right)+\phi\left(\rho_{j}\right)\right), \forall j \notin \mathcal{S}_{u}(\mathbf{x}),
$$


which combined with (60) gives the inequality

$$
\mu_{u}\left(\mathbf{x}_{-u}\right) \leq \pi_{j}\left(\rho_{j} \phi^{\prime}\left(\rho_{j}\right)+\phi\left(\rho_{j}\right)\right), \forall j \in \mathcal{S}
$$

Let $f_{j}:[0,1) \rightarrow\left[c_{j}, \infty\right)$ be defined by $f_{j}(x):=\pi_{j}\left(x \phi^{\prime}(x)+\phi(x)\right)$. Note that $f$ is increasing and nonnegative, and hence invertible. The inverse on $f_{j}$ is defined on $\left[c_{j}, \infty\right)$. Let us define $h_{j}:[0, \infty) \rightarrow[0,1)$ in the following way :

$$
h_{j}(x)=\left\{\begin{array}{ccc}
f_{J}^{-1}(x) & \text { if } & x \in\left[c_{j}, \infty\right) \\
0 & \text { if } & x \in\left[0, c_{j}\right) .
\end{array}\right.
$$

The function $h_{j}$ is continuous and non-decreasing. Further, from (61),

$$
h_{j}\left(\mu_{u}\left(\mathbf{x}_{-u}\right)\right) \leq \rho_{j}=\frac{\sum_{k} z_{k, j}}{r_{j}} .
$$

Summing over all the links, we obtain the following functional inequality on $\mu_{u}\left(\mathbf{x}_{-u}\right)$ :

$$
\bar{h}\left(\mu_{u}\left(\mathbf{x}_{-u}\right)\right):=\sum_{j} r_{j} h_{j}\left(\mu_{u}\left(\mathbf{x}_{-u}\right)\right) \leq \sum_{k} \sum_{j} z_{k, j}=\sum_{i} \lambda_{i}
$$

that is $\mu_{u}\left(\mathbf{x}_{-u}\right)$ is such that the above inequality is satisfied. A bound on $\mu_{u}\left(\mathbf{x}_{-u}\right)$ itself can now be obtained by making use of the following observations. Since $h_{j}$ is continuous and non-decreasing for all $j \in \mathcal{S}, \bar{h}$ is continuous and non-decreasing. It has $[0, \infty)$ as its domain and $\left[0, \sum_{j} r_{j}\right)$ as its image. Further, $\lim _{x \rightarrow \infty} \bar{h}(x)=\sum_{j} r_{j}$. From the stability condition, $\sum_{i} \lambda_{i}<\sum_{j} r_{j}$. Using these properties and (62), we can conclude that $\mu_{u}\left(\mathbf{x}_{-u}\right) \leq \mu_{\max }<\infty$.

It then follows from (59) that

$$
\rho_{j} \leq \beta_{j}=\phi^{-1}\left(\frac{\mu_{\max }}{\pi_{j}}\right), \quad \forall j \in \mathcal{S}_{u}(\mathbf{x}),
$$

and the upper bound $\beta_{j}$ depends neither upon $u$ nor upon $\mathbf{x}$. Moreover, $\phi$ is such that $x<\infty \Leftrightarrow \phi^{-1}(x)<1$, and hence $\beta_{j}<1$. By definition of $\mathcal{S}_{u}(\mathbf{x})$, we also have $\rho_{j}>0$ and thus $\beta_{j}>0$. Taking $\rho_{\max }=\max _{j \in \mathcal{S}_{u}(\mathbf{x})} \beta_{j}$ yields the proof.

Proof of Lemma 4. We note from (55) that, since $\frac{x_{u, k}}{r_{k}} \phi^{\prime \prime}\left(\rho_{k}\right) \geq 0$, we have $\theta_{k} \geq\left(\phi^{\prime}\left(\rho_{k}\right)+\right.$ $\left.\frac{x_{u, k}}{r_{k}} \phi^{\prime \prime}\left(\rho_{k}\right)\right) /\left(2 \phi^{\prime}\left(\rho_{k}\right)+2 \frac{x_{u, k}}{r_{k}} \phi^{\prime \prime}\left(\rho_{k}\right)\right)$, implying that

$$
\theta_{k} \geq \frac{1}{2}
$$

Since $\phi$ is increasing and convex,$\theta_{k}$ is an increasing function of $x_{u, k}$ (considering $\rho_{k}=\rho_{k}^{(u)}(\mathbf{x})$ as fixed), and since $x_{u, k} / r_{k} \leq \rho_{k}$, we also have the following inequality:

$$
\begin{aligned}
\theta_{k} & \leq \frac{\phi^{\prime}\left(\rho_{k}\right)+\rho_{k} \phi^{\prime \prime}\left(\rho_{k}\right)}{2 \phi^{\prime}\left(\rho_{k}\right)+\rho_{k} \phi^{\prime \prime}\left(\rho_{k}\right)} \\
& \leq 1-\frac{\phi^{\prime}\left(\rho_{k}\right)}{2 \phi^{\prime}\left(\rho_{k}\right)+\rho_{k} \phi^{\prime \prime}\left(\rho_{k}\right)}
\end{aligned}
$$

Since the numerator and the denominator of the fraction appearing on the right-hand side of (64) are strictly increasing in $\rho_{k}$, Lemma 6 implies that

$$
\theta_{k} \leq q=1-\frac{\phi^{\prime}(0)}{2 \phi^{\prime}\left(\rho_{\max }\right)+\rho_{\max } \phi^{\prime \prime}\left(\rho_{\max }\right)}<1
$$

A consequence of Theorem 3 and Lemma 4 . 
Proposition 2 The set $\mathcal{J}_{i}$ of Jacobian matrices is bounded.

Proof of Proposition 2. Consider a player $u \in \mathcal{C}$ and a point $\mathbf{x}$ where the best-response function $x^{(u)}$ is differentiable. Theorem 3 implies that

$$
\begin{aligned}
\left\|\mathrm{D} x^{(u)}(\mathbf{x})\right\|_{1} & \leq\|I\|_{1}+\left\|\mathrm{M}_{u}(\mathbf{x})\right\|_{1} \\
& \leq 1+\max _{m \in \mathcal{S}} \sum_{n \in \mathcal{S}_{u}(\mathbf{x})} \mid \theta_{m}\left(\gamma_{n}-\delta_{m}(n)\right) \|
\end{aligned}
$$

For $m \notin \mathcal{S}_{u}(\mathbf{x})$, we have

$$
\sum_{n \in \mathcal{S}_{u}(\mathbf{x})}\left|\theta_{m}\left(\gamma_{n}-\delta_{m}(n)\right)\right|=\theta_{m}<1
$$

while for $m \in \mathcal{S}_{u}(\mathbf{x})$, we have

$$
\begin{aligned}
\sum_{n \in \mathcal{S}_{u}(\mathbf{x})}\left|\theta_{m}\left(\gamma_{n}-\delta_{m}(n)\right)\right| & =\theta_{m}\left(\sum_{n \in \mathcal{S}_{u}(\mathbf{x}), n \neq m} \gamma_{n}+\left|\gamma_{m}-1\right|\right) \\
& =2 \theta_{m}\left(1-\gamma_{m}\right) \\
& <2 .
\end{aligned}
$$

With (66), (67) and (68), we obtain that $\left\|\mathrm{D} x^{(u)}(\mathbf{x})\right\|_{1}<3$ for all players $u \in \mathcal{C}$ and all points $\mathbf{x}$ where $x^{(u)}$ is differentiable. From its definition in (9), we thus conclude that the set $\mathcal{J}$ is bounded.

From the submultiplicativity of norms and relation (7), it follows that

Corollary 4 The set $\mathcal{J}$ is bounded.

\section{Proofs of results in Section 4.1}

Proof of Lemma 5. The proof is by induction. The claim is true for $n=1$. Given that the form is true for some $n$, it will be shown that the form holds for $n+1$. By definition,

$$
\begin{aligned}
\prod_{i=1}^{n+1} J_{i} & =J_{n+1} \prod_{i=1}^{n} J_{i} \\
& =\left(\begin{array}{cc}
0 & \Psi_{1} M_{1} \\
0 & \Psi_{2} M_{2} M_{1}
\end{array}\right) \cdot\left(\begin{array}{cc}
0 & \Psi_{3} X_{1}^{(2 n-1)} \\
0 & \Psi_{4} X_{2}^{(2 n)}
\end{array}\right) \\
& =\left(\begin{array}{cc}
0 & \Psi_{1} M_{1} \Psi_{3} X_{2}^{(2 n)} \\
0 & \Psi_{2} M_{2} M_{1} \Psi_{4} X_{2}^{(2 n)}
\end{array}\right)
\end{aligned}
$$

Since $M_{1} \in \mathcal{M}_{q}$ and $\Psi_{4}$ is a 0-1 diagonal matrix, it follows that $M_{1} \Psi_{4} \in \mathcal{M}_{q}$. Using the previous fact and the definition $\mathcal{M}_{q}^{(2 n)}$ and the fact that $X_{2}^{(2 n)} \in \mathcal{M}_{q}^{(2 n)}$, one can deduce that $\left(M_{1} \Psi_{3}\right) X_{2}^{(2 n)} \in \mathcal{M}_{q}^{(2(n+1)-1)}$, and $M_{2} M_{1} \Psi_{4} X_{2}^{(2 n)} \in \mathcal{M}_{q}^{(2(n+1))}$.

Proof of Corollary 3. Consider $M_{1}, M_{2} \ldots M_{n} \in \mathcal{M}_{q}$. Each matrix $M_{i}$ can be written as $M_{i}=$ $\left(\Gamma^{(i)} B-I\right) \Theta^{(i)}$, where $\theta_{\max }^{i}=\left\|\Theta^{(i)}\right\|_{1} \leq q$. From theorem 5, we thus obtain $\rho\left(M_{n} M_{n-1} \ldots M_{1}\right)^{\frac{1}{n}} \leq q$. As a consequence,

$$
\sup _{M_{1}, \ldots, M_{n} \in \mathcal{M}_{q}} \rho\left(\prod_{i=1}^{n} M_{i}\right)^{\frac{1}{n}} \leq q
$$


Since $\mathcal{M}_{q}$ is bounded, its joint spectral radius and its generalized spectral radius coincide. From the definition in (11), we immediately obtain that $\rho\left(\mathcal{M}_{q}\right) \leq q$.

Proof of Theorem 6. Since $\mathcal{J}$ is bounded (see Corollary 4), and the Generalized spectral radius is equal to the Joint spectral radius of a bounded set of matrices, it suffices to prove that $\rho(\mathcal{J})<1$. From Lemma 5, we have

$$
\operatorname{det}\left(\prod_{i=1}^{n} J_{i}-\lambda I\right)=\operatorname{det}(-\lambda I) \operatorname{det}\left(\Psi_{2} X_{2}^{(2 n)}-\lambda I\right)=(-\lambda)^{S} \operatorname{det}\left(\Psi_{2} X_{2}^{(2 n)}-\lambda I\right),
$$

implying that $\lambda \neq 0$ is an eigenvalue of $\prod_{i=1}^{n} J_{i}$ if and only if it is an eigenvalue of $\Psi_{2} X_{2}^{(2 n)}$. Thus, $\rho\left(\prod_{i=1}^{n} J_{i}\right)=\rho\left(\Psi_{2} X_{2}^{(2 n)}\right)$. Further,

$$
\rho\left(\Psi_{2} X_{2}^{(2 n)}\right) \leq\left\|\Psi_{2} X_{2}^{(2 n)}\right\|_{1} \leq\left\|\Psi_{2}\right\|_{1}\left\|X_{2}^{(2 n)}\right\|_{1}=\left\|X_{2}^{(2 n)}\right\|_{1},
$$

and thus, since $X_{2}^{(2 n)} \in \mathcal{M}_{q}^{(2 n)}$,

$$
\rho\left(\prod_{i=1}^{n} J_{i}\right) \leq \sup _{M \in \mathcal{M}_{q}^{(2 n)}}\|M\|_{1}=\rho_{2 n}\left(\mathcal{M}_{q}\right)
$$

where the last equality is obtained using the definition of the Joint spectral radius (10). Let $\epsilon=\frac{1-q}{2}>0$. Since $\rho_{n}\left(\mathcal{M}_{q}\right)^{\frac{1}{n}} \rightarrow \rho\left(\mathcal{M}_{q}\right)$ as $n \rightarrow \infty$, there exists $N$ such that for all $n \geq N$,

$$
\rho\left(\prod_{i=1}^{n} J_{i}\right)^{\frac{1}{n}} \leq \rho\left(\mathcal{M}_{q}\right)+\epsilon \leq q+\frac{1-q}{2}=\frac{1+q}{2},
$$

where the last inequality follows from Corollary 3 . Since the right hand-side is independant of $J_{1}, \ldots, J_{n}$, we deduce that

$$
\sup _{J_{1}, \ldots, J_{n} \in \mathcal{J}} \rho\left(\prod_{i=1}^{n} J_{i}\right)^{\frac{1}{n}} \leq \frac{1+q}{2}, \quad \forall n \geq N,
$$

and, according to 11, it yields $\rho(\mathcal{J}) \leq \frac{1+q}{2}<1$.

\section{Proof of Theorem 5}

The main difficulty in proving Theorem 5 is that the matrices $M$ of $\mathcal{M}^{(k)}$ are neither positive nor negative. To circumvent this difficulty, we shall construct a positive or negative matrix $A$ such that $\rho(M) \leq \rho(A)$ and $\|A\|_{1} \leq \prod_{i=1}^{k} \theta_{\text {max }}^{i}$. Before showing how to construct such a matrix, we state two basic properties of the matrices in $\mathcal{M}^{(k)}$ in the following lemma.

Lemma 7 For any matrix $M \in \mathcal{M}^{(k)}$, the following two assertions hold:

(a) for each and every column $j, \sum_{i=1}^{S} m_{i, j}=0$,

(b) if $\lambda \neq 0$ is an eigenvalue of $M$ and if $\mathbf{x}$ is the associated eigenvector, then $\sum_{i=1}^{S} x_{i}=0$.

Proof. Let us first prove assertion (a). Consider $M \in \mathcal{M}^{(k)}$ and write $M$ as $M=(\Gamma B-I) \Theta Y$ with $Y \in \mathcal{M}^{(k-1)}$. Then, 


$$
\begin{aligned}
\left(\sum_{i} m_{i, 1}, \ldots, \sum_{i} m_{i, S}\right)=\mathbf{1}^{T} M & =\mathbf{1}^{T}(\Gamma B-I) \Theta Y \\
& =\left(\sum_{i} \gamma_{i}-1, \ldots, \sum_{i} \gamma_{i}-1\right) Y \\
& =\mathbf{0}^{T}
\end{aligned}
$$

which proves the result. Let us now prove assertion (b). Let $M \in \mathcal{M}^{(k)}$ be written in the form $M=$ $(\Gamma B-I) \Theta Y$ and consider $\lambda \in \sigma(M), \lambda \neq 0$ and $\mathbf{x} \neq \mathbf{0}$ such that $\lambda \mathbf{x}=M \mathbf{x}$. Multiplying on both sides by $\mathbf{1}^{T}$, we obtain

$$
\lambda \sum_{i=1}^{S} x_{i}=0=\mathbf{1}^{T} \mathbf{x}=\mathbf{1}^{T} M \mathbf{x}=0
$$

where the last equality follows from assertion (a). Since $\lambda \neq 0$, this implies that $\sum_{i=1}^{S} x_{i}=0$.

We will now use property (b) of Lemma 7 to show that, for any matrix $M \in \mathcal{M}^{(k)}$, if we choose the matrix $A$ to be of the form $A=D B+M$, where $D$ is any diagonal matrix, then $\rho(M) \leq \rho(A)$.

Lemma 8 For any matrix $M \in \mathcal{M}^{(k)}$ and for any diagonal matrix $D, \rho(M) \leq \rho(D B+M)$.

Proof. Let $\lambda \neq 0$ be an eigenvalue of $M$ and $\mathbf{x}$ be the associated eigenvector. We have

$$
(D B+M) \mathbf{x}=D B \mathbf{x}+\lambda \mathbf{x}=\left(\sum_{i} x_{i}\right) D \mathbf{1}+\lambda \mathbf{x}=\lambda \mathbf{x},
$$

where the last equality is obtained using property (b) of Lemma 7 . Since this can be done for all non-zero eigenvalues of $M$, we conclude that $\sigma(M)-\{0\} \subset \sigma(D B+M)$. This clearly implies that

$$
\max _{\lambda \in \sigma(M)}|\lambda| \leq \max _{\lambda \in \sigma(D B+M)}|\lambda|
$$

i.e., $\rho(M) \leq \rho(D B+M)$.

Given a matrix $M \in \mathcal{M}^{(k)}$, we shall now consider two specific choices of the diagonal matrix $D$ : the first choice allows to obtain a matrix $A \geq 0$ such that $\rho(M) \leq \rho(A)$, while the second one produces a matrix $A \leq 0$ with the same property. Since the two choices lead to a positive or negative matrix $A$, the evaluation of $\|A\|_{1}$ is greatly simplified, allowing to obtain useful upper bounds on $\rho(M)$. These bounds are proven in the following proposition.

Proposition 3 For any matrix $M \in \mathcal{M}^{(k)}$, the two following inequalities on $\rho(M)$ are valid:

$$
\begin{aligned}
\rho(M) & \leq-\sum_{i=1}^{S} \min _{1 \leq k \leq S}\left(m_{i, k}\right), \\
\rho(M) & \leq \sum_{i=1}^{S} \max _{1 \leq k \leq S}\left(m_{i, k}\right),
\end{aligned}
$$

Proof. Let us first consider the diagonal matrix $D$ defined as

$$
D=-\operatorname{diag}\left(\min _{k}\left(m_{1, k}\right), \min _{k}\left(m_{2, k}\right), \ldots, \min _{k}\left(m_{S, k}\right)\right),
$$

and consider the matrix $A=D B+M$. Since $a_{i, j}=m_{i, j}-\min _{k}\left(m_{i, k}\right), \forall i, j$, we have $A \geq 0$. We know from Lemma 8 that $\rho(M) \leq \rho(A) \leq\|A\|_{1}$. Hence 


$$
\begin{aligned}
\rho(M) & \leq \max _{1 \leq j \leq S}\left(\sum_{i} a_{i, j}\right), \\
& \leq \max _{1 \leq j \leq S}\left(\sum_{i} m_{i, j}-\sum_{i} \min _{k}\left(m_{i, k}\right)\right), \\
& \leq-\sum_{i} \min _{k}\left(m_{i, k}\right),
\end{aligned}
$$

where the last inequality is obtained using property (a) of Lemma 7

To prove the second inequality, we now define the matrix D as follows

$$
D=-\operatorname{diag}\left(\max _{k}\left(m_{1, k}\right), \max _{k}\left(m_{2, k}\right), \ldots, \max _{k}\left(m_{S, k}\right)\right),
$$

and obtain a matrix $A=D B+M \leq 0$ since $a_{i, j}=m_{i, j}-\max _{k}\left(m_{i, k}\right), \forall i, j$. Again, using $\rho(M) \leq \rho(A) \leq$ $\|A\|_{1}$, we obtain

$$
\begin{aligned}
\rho(M) & \leq \max _{1 \leq j \leq S}\left(-\sum_{i} a_{i, j}\right), \\
& \leq \max _{1 \leq j \leq S}\left(\sum_{i} \max _{k}\left(m_{i, k}\right)-\sum_{i} m_{i, j}\right), \\
& \leq \sum_{i} \max _{k}\left(m_{i, k}\right),
\end{aligned}
$$

and both inequalities on $\rho(M)$ are proved.

We will now prove that we can recursively obtain upper bounds on the terms appearing on the right-hand sides of (70) and (71).

Lemma 9 Let the matrix $M$ be in $\mathcal{M}^{(k)}$ and let $X \in \mathcal{M}^{(k-1)}, \Theta \in \mathcal{D}^{+}$and $\Gamma \in \mathcal{G}$ be such that $M=$ $X(\Gamma B-I) \Theta$. Then

$$
\begin{aligned}
-\sum_{i=1}^{S} \min _{1 \leq j \leq S}\left(m_{i, j}\right) & \leq \theta_{\max } \sum_{i=1}^{S} \max _{1 \leq j \leq S}\left(x_{i, j}\right), \\
\sum_{i=1}^{S} \max _{1 \leq j \leq S}\left(m_{i, j}\right) & \leq-\theta_{\max } \sum_{i=1}^{S} \min _{1 \leq j \leq S}\left(x_{i, j}\right),
\end{aligned}
$$

where $\theta_{\max }=\max _{i} \theta_{i}$

Proof. We have $m_{i, j}=\theta_{j}\left(\sum_{k} x_{i, k} \gamma_{k}-x_{i, j}\right), \forall i, j$. Since $\max _{j}\left(x_{i, j}\right) \geq \sum_{k} x_{i, k} \gamma_{k}$ for all $i$, we have

$$
m_{i, j} \geq \theta_{j}\left(\sum_{k} x_{i, k} \gamma_{k}-\max _{j}\left(x_{i, j}\right)\right) \geq \theta_{\max }\left(\sum_{k} x_{i, k} \gamma_{k}-\max _{j}\left(x_{i, j}\right)\right),
$$

for all $i, j=1, \ldots, S$, and thus $\min _{j}\left(m_{i, j}\right) \geq \theta_{\max }\left(\sum_{k} x_{i, k} \gamma_{k}-\max _{j}\left(x_{i, j}\right)\right)$. As a consequence 


$$
\begin{aligned}
\sum_{i} \min _{j}\left(m_{i, j}\right) & \geq \theta_{\max }\left(\sum_{i} \sum_{k} x_{i, k} \gamma_{k}-\sum_{i} \max _{j}\left(x_{i, j}\right)\right), \\
& \geq \theta_{\max }\left(\sum_{k}\left(\sum_{i} x_{i, k}\right) \gamma_{k}-\sum_{i} \max _{j}\left(x_{i, j}\right)\right),
\end{aligned}
$$

and since $\sum_{i} x_{i, k}=0$ for all $k$ according to property (a) of Lemma 7 it yields

$$
\sum_{i} \min _{j}\left(m_{i, j}\right) \geq-\theta_{\max } \sum_{i} \max _{j}\left(x_{i, j}\right)
$$

which proves that $-\sum_{i} \min _{j}\left(m_{i, j}\right) \leq \theta_{\max } \sum_{i} \max _{j}\left(x_{i, j}\right)$, as claimed.

The proof of the second inequality is similar. We observe that

$$
m_{i, j} \leq \theta_{j}\left(\sum_{k} x_{i, k} \gamma_{k}-\min _{j}\left(x_{i, j}\right)\right) \leq \theta_{\max }\left(\sum_{k} x_{i, k} \gamma_{k}-\min _{j}\left(x_{i, j}\right)\right),
$$

for all $i, j=1, \ldots, S$, and thus $\max _{j}\left(m_{i, j}\right) \leq \theta_{\max }\left(\sum_{k} x_{i, k} \gamma_{k}-\min _{j}\left(x_{i, j}\right)\right)$. It yields

$$
\begin{aligned}
\sum_{i} \max _{j}\left(m_{i, j}\right) & \leq \theta_{\max }\left(\sum_{i} \sum_{k} x_{i, k} \gamma_{k}-\sum_{i} \min _{j}\left(x_{i, j}\right)\right) \\
& \leq \theta_{\max }\left(\sum_{k}\left(\sum_{i} x_{i, k}\right) \gamma_{k}-\sum_{i} \min _{j}\left(x_{i, j}\right)\right) \\
& \leq-\theta_{\max } \sum_{i} \min _{j}\left(x_{i, j}\right),
\end{aligned}
$$

as claimed.

We are now in position to prove Theorem 5

Proof of Theorem 5. Consider a matrix $M=\prod_{i=1}^{k}\left(\Gamma^{(i)} B-I\right) \Theta^{(i)}$ in $\mathcal{M}^{(k)}$. Define the matrices $X^{(n)}=\prod_{i=1}^{n}\left(\Gamma^{(i)} B-I\right) \Theta^{(i)}$ for $n=1,2, \ldots, k$. Note that $X^{(n)} \in \mathcal{M}^{(n)}$, that $M=X^{(k)}$ and that $X^{(n)}=X^{(n-1)}\left(\Gamma^{(n)} B-I\right) \Theta^{(n)}$ for $1<n \leq k$.

We have $X^{(1)}=\left(\Gamma^{(1)} B-I\right) \Theta^{(1)}$. With (71) we have $\rho\left(X^{(1)}\right) \leq \sum_{i=1}^{S} \max _{1 \leq k \leq S}\left(x_{i, k}^{(1)}\right)$. However

$$
\begin{aligned}
\sum_{i=1}^{S} \max _{1 \leq k \leq S}\left(x_{i, k}^{(1)}\right) & \leq \sum_{i=1}^{S} \max \left(\left(\gamma_{i}^{(1)}-1\right) \theta_{i}^{(1)}, \max _{k \neq i}\left(\gamma_{i}^{(1)} \theta_{k}^{(1)}\right)\right) \\
& \leq \theta_{\max }^{(1)} \sum_{i=1}^{S} \gamma_{i}^{(1)}=\theta_{\max }^{(1)}
\end{aligned}
$$

from wich we conclude that $\rho\left(X^{(1)}\right) \leq \theta_{\max }^{(1)}$. If $k=1$, we have $M=X^{(1)}$ and thus $\rho(M) \leq \theta_{\max }^{(1)}$. For $k>1$, we consider separately the case when it is even and the case when it is odd. If $k$ is even, Proposition 3 states that

$$
\rho(M) \leq-\sum_{i=1}^{S} \min _{1 \leq k \leq S}\left(m_{i, k}\right),
$$

and the repeated application of Lemma 9 yields 


$$
\begin{aligned}
\rho(M) & \leq \theta_{\max }^{(k)} \sum_{i=1}^{S} \max _{1 \leq j \leq S}\left(x_{i, j}^{(k-1)}\right) \\
& \leq-\theta_{\max }^{(k)} \theta_{\max }^{(k-1)} \sum_{i=1}^{S} \min _{1 \leq j \leq S}\left(x_{i, j}^{(k-2)}\right) \\
& \vdots \\
& \leq \prod_{i=2}^{k} \theta_{\max }^{(i)} \sum_{i=1}^{S} \max _{1 \leq k \leq S}\left(x_{i, k}^{(1)}\right),
\end{aligned}
$$

and we conclude with (77) that $\rho(M) \leq \prod_{i=1}^{k} \theta_{\text {max }}^{(i)}$. If on the contrary $k$ is odd, we use the second inequality in Proposition 3 to obtain

$$
\rho(M) \leq \sum_{i=1}^{S} \max _{1 \leq k \leq S}\left(m_{i, k}\right) .
$$

Applying again repeatedly Lemma 9 yields

$$
\begin{aligned}
\rho(M) & \leq-\theta_{\max }^{(k)} \sum_{i=1}^{S} \min _{1 \leq j \leq S}\left(x_{i, j}^{(k-1)}\right) \\
& \leq \theta_{\max }^{(k)} \theta_{\max }^{(k-1)} \sum_{i=1}^{S} \max _{1 \leq j \leq S}\left(x_{i, j}^{(k-2)}\right) \\
& \vdots \\
& \leq \prod_{i=2}^{k} \theta_{\max }^{(i)} \sum_{i=1}^{S} \max _{1 \leq k \leq S}\left(x_{i, k}^{(1)}\right),
\end{aligned}
$$

and with (77) it proves that $\rho(M) \leq \prod_{i=1}^{k} \theta_{\max }^{(i)}$. We therefore conclude that $\rho(M) \leq \prod_{i=1}^{k} \theta_{\max }^{(i)}$ for all matrices $\mathcal{M}^{(k)}$, and for all $k \geq 1$.

We prove below that there exist some matrices in $\mathcal{M}^{(k)}$ for which the upper bound on the spectral radius of Theorem 5 is tight.

Lemma 10 For any $k \geq 1$, there exists $M \in \mathcal{M}^{(k)}$ such that $\rho(M)=\prod_{i=1}^{k} \theta_{\max }^{(i)}$.

Proof. Consider a matrix $M=\prod_{i=1}^{k}\left(\Gamma^{(i)} B-I\right) \Theta^{(i)} \in \mathcal{M}^{(k)}$ such that $\Theta^{(i)}=\theta_{\max }^{(i)} I$ for all $1 \leq i \leq k$. Obviously, $M=\left(\prod_{i=1}^{k} \theta_{\max }^{(i)}\right) \prod_{i=1}^{k}\left(\Gamma^{(i)} B-I\right)$. Observe now that for all $m, n$

$$
\Gamma^{(m)} B \Gamma^{(n)} B=\left(\sum_{i} \gamma_{i}^{(n)}\right) \Gamma^{(m)} B=\Gamma^{(m)} B
$$

which implies that

$$
\begin{aligned}
\left(\Gamma^{(m)} B-I\right)\left(\Gamma^{(n)} B-I\right) & =\Gamma^{(m)} B \Gamma^{(n)} B-\Gamma^{(m)} B-\Gamma^{(n)} B+I \\
& =\Gamma^{(m)} B-\Gamma^{(m)} B-\Gamma^{(n)} B+I \\
& =-\left(\Gamma^{(n)} B-I\right) .
\end{aligned}
$$


Hence

$$
\prod_{i=1}^{k}\left(\Gamma^{(i)} B-I\right)=(-1)^{k}\left(\Gamma^{(k)} B-I\right),
$$

and thus we obtain $M=(-1)^{k}\left(\prod_{i=1}^{k} \theta_{\max }^{(i)}\right)\left(\Gamma^{(k)} B-I\right)$, which implies that $\rho(M)=\left(\prod_{i=1}^{k} \theta_{\max }^{(i)} \rho\left(\Gamma^{(k)} B-I\right)\right.$.

We note that $\Gamma^{(k)} B$ is a matrix of rank 1 , since all its columns are the same. Moreover, the sum of each column is 1 . Thus, the spectrum of $\Gamma^{(k)} B$ is $\{1,0,0 \ldots, 0\}$, which implies that the spectrum of $\Gamma^{(k)} B-I$ is $\sigma\left(\Gamma^{(k)} B-I\right)=\{0,-1,-1 \ldots-1\}$. We conclude that $\rho\left(\Gamma^{(k)} B-I\right)=1$, which implies that $\rho(M)=\left(\prod_{i=1}^{k} \theta_{\max }^{(i)}\right)$.

\section{E Equivalence of functions}

Let $\psi$ be a function satisying assumptions $\left(B_{1}\right)-\left(B_{3}\right)$. If the link cost function is $\psi$, then the player $i$ will solve the following optimization problem:

$$
\begin{aligned}
& \operatorname{minimize} T_{i}\left(\mathbf{x}, \mathbf{x}_{-i}\right)=\sum_{j \in \mathcal{S}} c_{j} x_{i, j} \psi\left(y_{j}\right) \\
& \text { subject to } \\
& \qquad \sum_{j \in \mathcal{S}} x_{i, j}=\lambda_{i}, \\
& \qquad y_{j}=x_{i, j}+\sum_{k \neq i} x_{k, j}, \quad \forall j \in \mathcal{S}, \\
& x_{i, j} \geq 0, \quad \forall j \in \mathcal{S},
\end{aligned}
$$

Note that there is no capacity associated with a link, and there is no constraint of the type $y_{j}<r_{j}$.

Let $r>\bar{\lambda}$ be a constant. Define the function $\phi$ as

$$
\phi(x)= \begin{cases}\psi(r x) & \text { if } x \leq \rho \\ \psi(r x)+\frac{1}{1-x}+\frac{1-x}{(1-\rho)^{2}}-\frac{2}{1-\rho} & \text { if } \rho<x<1\end{cases}
$$

where $\rho=\frac{\bar{\lambda}}{r}$. It can be verified that the $\phi$ satisaties assumptions $\left(A_{1}\right)-\left(A_{3}\right)$.

We now show that the best-response of a player $i$ when it solves (BRpsi- $i$ ) is the solution of (BR-i) with $\phi$ as in (85), and $r_{j}=r, \forall j \in \mathcal{S}$. If $\mathbf{z} \in \mathcal{X}_{i}$ is a solution of (BR-i), then according to the KKT conditions:

$$
\begin{aligned}
\frac{c_{j}}{r}\left[\phi\left(\frac{y_{j}}{r}\right)+z_{j} \phi^{\prime}\left(\frac{y_{j}}{r}\right) \frac{1}{r}\right] & =\mu_{i} \quad \text { if } z_{j}>0, \\
\frac{c_{j}}{r} \phi\left(\frac{y_{j}}{r}\right) & >\mu_{i} \quad \text { if } z_{j}=0 .
\end{aligned}
$$

Using the relation (85), the above conditions can be replaced by:

$$
\begin{aligned}
c_{j}\left[\psi\left(y_{j}\right)+z_{j} \psi^{\prime}\left(y_{j}\right)\right] & =r \mu_{i} & & \text { if } z_{j}>0, \\
c_{j} \psi\left(y_{j}\right) & >r \mu_{i} & & \text { if } z_{j}=0 .
\end{aligned}
$$

where we have used the fact that, $\forall j \in \mathcal{S}, y_{j} / r \leq \bar{\lambda} / r=\rho$.

It can be verified that (86)-(87) are the KKT conditions for (BRpsi-i $)$. Hence, both (BR-i and (BRpsi-i have the same best response dynamics. 


\section{F Proof of Theorem 7}

Proof. We shall show that the product of Jacobian matrices over $n$ rounds goes to 0 as $n \rightarrow \infty$. This shows that their JSR is less than 1 , and hence best-response converges.

First we shall show this for the three player game as the proof follows the same steps for any number of players. Omitting the multiplier $\Psi$, the one-round Jacobian matrix for three players has the form :

$$
J^{(1)}=J_{3} J_{2} J_{3}=\left(\begin{array}{ccc}
0 & M_{1} & M_{1} \\
0 & M_{2} M_{1} & M_{2}+M_{2} M_{1} \\
0 & M_{3}\left(M_{2} M_{1}+M_{1}\right) & \Psi M_{3}\left(M_{2}+M_{2} M_{1}+M_{1}\right)
\end{array}\right) .
$$

Note that $M_{v} M_{u}=\left(\Gamma_{v} B-I\right) \Theta_{v}\left(\Gamma_{u} B-I\right) \Theta_{u}=\theta^{2}\left(\Gamma_{v} B-I\right)\left(\Gamma_{u} B-I\right)$ where $\theta=1 / 2$.

Denote $\Gamma_{u} B-I=H_{u}$, then $M_{v} M_{u}=-\theta^{2} H_{u}$, and the Jacobian matrix for one round, is as follows

$$
J^{(1)}=\left(\begin{array}{ccc}
0 & H_{1}^{(1)} \theta & H_{1}^{(1)} \theta \\
0 & -H_{1}^{(1)} \theta^{2} & H_{2}^{(1)} \theta-H_{1}^{(1)} \theta^{2} \\
0 & H_{1}^{(1)} \theta^{3}-H_{1}^{(1)} \theta^{2} & -H_{2}^{(1)} \theta^{2}+H_{1}^{(1)} \theta^{3}-H_{1}^{(1)} \theta^{2}
\end{array}\right)=\left(\begin{array}{ccc}
0 & H_{1}^{(1)} \theta & H_{1}^{(1)} \theta \\
0 & -H_{1}^{(1)} \theta^{2} & -H_{1}^{(1)} \theta^{2}+H_{2}^{(1)} \theta \\
0 & -H_{1}^{(1)} \theta^{2}+H_{1}^{(1)} \theta^{3} & H_{1}^{(1)} \theta^{3}-H_{1}^{(1)} \theta^{2}-H_{2}^{(1)} \theta^{2}
\end{array}\right) .
$$

Note here that for any round $n,\left(H_{u}^{(n)}\right)^{2}=-H_{u}^{(n)}$ and for different rounds $n, m, H_{v}^{(m)} H_{u}^{(n)}=-H_{u}^{(n)}$. With notation $p_{i, j}^{(n)}(\theta)$ and $q_{i, j}^{(n)}(\theta)$, or more simply $p_{i, j}^{(n)}$ and $q_{i, j}^{(n)}$, for polynomial coefficients of $H_{1}^{(n)}$ and $H_{2}^{(n)}$, respectively, the Jacobian matrix $J^{(n)}$ after $n$ rounds, it will take the following form

$$
J^{(n)}=\left(\begin{array}{ccc}
0 & H_{1}^{(1)} p_{1,2}^{(n)}+H_{2}^{(1)} q_{1,2}^{(n)} & H_{1}^{(1)} p_{1,3}^{(n)}+H_{2}^{(1)} q_{1,3}^{(n)} \\
0 & H_{1}^{(1)} p_{2,2}^{(n)}+H_{2}^{(1)} q_{2,2}^{(n)} & H_{1}^{(1)} p_{2,3}^{(n)}+H_{2}^{(1)} q_{2,3}^{(n)} \\
0 & H_{1}^{(1)} p_{3,2}^{(n)}+H_{2}^{(1)} q_{3,2}^{(n)} & H_{1}^{(1)} p_{3,3}^{(n)}+H_{2}^{(1)} q_{3,3}^{(n)}
\end{array}\right)
$$

To find recurrence relation between the polynomial coefficients in successive rounds, write

$$
\begin{aligned}
& J^{(n+1)}=\left(\begin{array}{ccc}
0 & H_{1}^{(n+1)} \theta & H_{1}^{(n+1)} \theta \\
0 & -H_{1}^{(n+1)} \theta^{2} & -H_{1}^{(n+1)} \theta^{2}+H_{2}^{(n+1)} \theta \\
0 & H_{1}^{(n+1)} \theta^{3}-H_{1}^{(n+1)} \theta^{2} & H_{1}^{(n+1)} \theta^{3}-H_{1}^{(n+1)} \theta^{2}-H_{2}^{(n+1)} \theta^{2}
\end{array}\right) J^{(n)} \\
& =\left(\begin{array}{ccc}
0 & H_{1}^{(n+1)} \theta & H_{1}^{(n+1)} \theta \\
0 & -H_{1}^{(n+1)} \theta^{2} & -H_{1}^{(n+1)} \theta^{2}+H_{2}^{(n+1)} \theta \\
0 & H_{1}^{(n+1)} \theta^{3}-H_{1}^{(n+1)} \theta^{2} & H_{1}^{(n+1)} \theta^{3}-H_{1}^{(n+1)} \theta^{2}-H_{2}^{(n+1)} \theta^{2}
\end{array}\right)\left(\begin{array}{ccc}
0 & H_{1}^{(1)} p_{1,2}^{(n)}+H_{2}^{(1)} q_{1,2}^{(n)} & H_{1}^{(1)} p_{1,3}^{(n)}+H_{2}^{(1)} q_{1,3}^{(n)} \\
0 & H_{1}^{(1)} p_{2,2}^{(n)}+H_{2}^{(1)} q_{2,2}^{(n)} & H_{1}^{(1)} p_{2,3}^{(n)}+H_{2}^{(1)} q_{2,3}^{(n)} \\
0 & H_{1}^{(1)} p_{3,2}^{(n)}+H_{2}^{(1)} q_{3,2}^{(n)} & H_{1}^{(1)} p_{3,3}^{(n)}+H_{2}^{(1)} q_{3,3}^{(n)}
\end{array}\right) .
\end{aligned}
$$

One can then deduce the following recursive expressions for the vectors of polynomial coefficients in the second column.

$$
\left(\begin{array}{l}
p_{1, j}^{(n+1)} \\
p_{2, j}^{(n+1)} \\
p_{3, j}^{(n+1)}
\end{array}\right)=\left(\begin{array}{ccc}
0 & -\theta & -\theta \\
0 & \theta^{2} & \theta^{2}-\theta \\
0 & -\theta^{3}+\theta^{2} & -\theta^{3}+2 \theta^{2}
\end{array}\right)\left(\begin{array}{l}
p_{1, j}^{(n)} \\
p_{2, j}^{(n)} \\
p_{3, j}^{(n)}
\end{array}\right) \text {, and }\left(\begin{array}{l}
q_{1, j}^{(n+1)} \\
q_{2, j}^{(n+1)} \\
q_{3, j}^{(n+1)}
\end{array}\right)=\left(\begin{array}{ccc}
0 & -\theta & -\theta \\
0 & \theta^{2} & \theta^{2}-\theta \\
0 & -\theta^{3}+\theta^{2} & -\theta^{3}+2 \theta^{2}
\end{array}\right)\left(\begin{array}{l}
q_{1, j}^{(n)} \\
q_{2, j}^{(n)} \\
q_{3, j}^{(n)}
\end{array}\right) \text {. }
$$

A similar relation can be deduced for the vector of polynomials in the third column.

If it can be shown that the spectral radius of the matrix

$$
A_{3}=\left(\begin{array}{ccc}
0 & -\theta & -\theta \\
0 & \theta^{2} & \theta^{2}-\theta \\
0 & -\theta^{3}+\theta^{2} & -\theta^{3}+2 \theta^{2}
\end{array}\right)
$$

is less than 1, then we can conclude that the any product of Jacobian matrices will go to 0 in any norm as $n \rightarrow \infty$, and thus conclude that the JSR of $\mathcal{J}$ is smaller than 1 .

For a $K$ player game, it turns out that the matrix $A_{K}$ has the form

$$
\left[A_{K}\right]_{i, j}= \begin{cases}(1-\theta)^{i-1} & \text { for } j>i, \\ (1-\theta)^{i-1}-(1-\theta)^{i-j} & \text { for } j \leq i .\end{cases}
$$


which is expanded form is

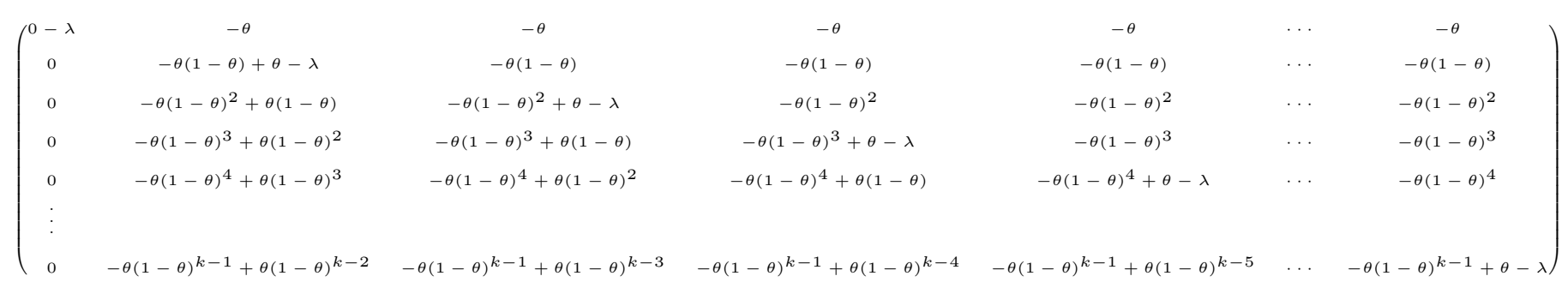

In Proposition 4 stated just after this proof, it is shown that the spectral radius of $A_{K}$ is less than $\theta$ which is less than 1 . We can thus conclude that the product of Jacobians will tend to 0 as $n \rightarrow \infty$, and hence the best-response will converge.

Proposition 4 The spectral radius of the matrix $A_{K}$ defined in (88) is less than $\theta$.

Proof. We shall show that the zeros of $\operatorname{det}\left(A_{K}-\lambda I\right)$ are in the unit circle. Transform the $A_{K}-\lambda I$ by multiplying each row $i$ by $-(1-\theta)$ and adding it to row $i+1$, for $i=K-1, K-2, \ldots, 1$, to get

$$
\operatorname{det}\left(A_{K}-\lambda I\right)=\operatorname{det}\left(\begin{array}{ccccccc}
-\lambda & -\theta & -\theta & -\theta & \cdots & -\theta & -\theta \\
\lambda(1-\theta) & \theta-\lambda & 0 & 0 & \cdots & 0 & 0 \\
0 & \lambda(1-\theta) & \theta-\lambda & 0 & \cdots & 0 & 0 \\
0 & 0 & \lambda(1-\theta) & \theta-\lambda & \cdots & 0 & 0 \\
0 & 0 & 0 & \lambda(1-\theta) & \cdots & 0 & 0 \\
\vdots & & & & & & \\
0 & 0 & 0 & 0 & \ldots & \lambda(1-\theta) & \theta-\lambda
\end{array}\right) .
$$

Computing the determinant along the last column, one obtains the polynomial

$$
\operatorname{det}\left(A_{K}-\lambda I\right)=(-1)^{k}\left[(\lambda-\theta)^{k}+\theta \sum_{i=0}^{k-1}(\lambda-\theta)^{k-1-i} \lambda^{i}(1-\theta)^{i}\right] .
$$

Denote the expression in the square brackets by $\mathcal{P}(\lambda)$. For $\lambda \neq 1$, after some algebra, we obtain

$$
\mathcal{P}(\lambda)=\frac{(1-\theta)^{k}}{1-\lambda}\left[\lambda^{k}-\frac{\lambda(\lambda-\theta)^{k}}{(1-\theta)^{k}}\right]
$$

Since $0<\theta<1$, for $\lambda<0, \mathcal{P}(\lambda)$ is negative for even $k$ and positive for odd $k$.

For $\lambda>1$, the denominator $(1-\lambda)$ is negative. Note that for $\lambda>1, \theta<\theta \lambda<\lambda$ and then $0<\lambda-\theta \lambda<$ $\lambda-\theta$. Then for the expression in the numerator the following holds, $\lambda^{k}(1-\theta)^{k}-\lambda(\lambda-\theta)^{k}=(\lambda-\theta \lambda)^{k}-\lambda(\lambda-\theta)^{k}<(\lambda-\theta \lambda)^{k}-(\lambda-\theta)^{k}<0$. Hence $\mathcal{P}(\lambda)>0$.

For $\theta<\lambda<1$, the denominator $(1-\lambda)$ is positive. Note that for $0<\lambda<\theta, 0<\theta \lambda<\theta<\lambda<1$ and then $0<\lambda-\theta<\lambda-\theta \lambda$. Then for the expression in the numerator, $\lambda^{k}(1-\theta)^{k}-\lambda(\lambda-\theta)^{k}=(\lambda-\theta \lambda)^{k}-\lambda(\lambda-\theta)^{k}>(\lambda-\theta \lambda)^{k}-(\lambda-\theta)^{k}>0$.

Moreover, $\mathcal{P}(1)>0$.

Thus, the zeros of the function $\mathcal{P}(\lambda)$ are in $[0, \theta]$. 